\title{
A LEVEL SET METHOD IN SHAPE AND TOPOLOGY OPTIMIZATION FOR VARIATIONAL INEQUALITIES
}

\author{
Piotr FUlMAŃSKI *, ANTOINe LAURAIN **, JeAn-FranCoIS SCHEID **, \\ JAN SOKOŁOWSKI ${ }^{* *}$ \\ ${ }^{*}$ Faculty of Mathematics, University of Łódz, \\ ul. Banacha 22, 90-232 Łódź, Poland \\ e-mail: fulmanp@imul.math.uni.lodz.pl \\ ** Institut Elie Cartan, Laboratoire de Mathématiques, Université Henri Poincaré Nancy I, \\ B.P. 239, 54506 Vandoeuvre-lès-Nancy Cedex, France \\ e-mail: antoine.laurain@iecn.u-nancy.fr \\ e-mail: jean-francois.scheid@iecn-u.nancy.fr \\ e-mail: sokolows@iecn.u-nancy.fr
}

\begin{abstract}
The level set method is used for shape optimization of the energy functional for the Signorini problem. The boundary variations technique is used in order to derive the shape gradients of the energy functional. The conical differentiability of solutions with respect to the boundary variations is exploited. The topology modifications during the optimization process are identified by means of an asymptotic analysis. The topological derivatives of the energy shape functional are employed for the topology variations in the form of small holes. The derivation of topological derivatives is performed within the framework proposed in (Sokołowski and Żochowski, 2003). Numerical results confirm that the method is efficient and gives better results compared with the classical shape optimization techniques.
\end{abstract}

Keywords: shape optimization, topological derivative, level set method, variational inequality, asymptotic analysis

\section{Introduction}

In the present paper a numerical method for shape and topology optimization of the energy functional for the Signorini problem is proposed. The method requires the evaluation of the shape gradients and topological derivatives of the functional in question, and the level set method is used for the evolution of geometrical domains.

The Hamilton-Jacobi nonlinear hyperbolic equation models the evolution of the level set function. The normal speed of the moving boundaries is determined from the shape gradients obtained for the energy functional (Sokołowski and Zolesio, 1992).

During the optimization process, the topology changes are defined by the topological derivatives of the energy functional. Small holes are injected into the actual geometrical domain with the centers at the points determined by the maximization of the topological derivatives.

We also provide arguments which allow us to determine the topological derivatives. To this end, the domain decomposition technique is applied. The proof of the asymptotic expansion of the Steklov-Poincaré opera- tor used in such a technique is given in Appendix. The technique for such an analysis is proposed in (Masmoudi, 2002; Sokołowski and Żochowski, 2005a).

Singular perturbations of domains in the framework of shape optimization are studied in (Allaire et al., 2005; Jackowska et al., 2002; Jackowska et al., 2003; Maz'ya et al., 2000; Nazarov, 1999; Nazarov and Sokołowski, 2003b; Nazarov and Sokołowski, 2003a; Nazarov and Sokołowski, 2004a; Nazarov and Sokołowski, 2004b; Nazarov et al., 2005; Sokołowski and Żochowski, 1999; Sokołowski and Żochowski, 2001; Sokołowski and Żochowski, 2003). The construction of the asymptotic expansion for the Steklov-Poincaré operator is given in (Sokołowski and Żochowski, 2005b).

\section{Signorini Problem}

We introduce the model problem. Let $U$ and $V$ be two bounded open subsets of $\mathbb{R}^{2}$ such that $V \subset \subset U$. For any open set $\omega \subset \mathbb{R}^{2}$, we denote by $\# \bar{\omega}$ the number of 
connected components of $\bar{\omega}$ and we consider the set of admissible domains

$$
\mathcal{O}_{k}=\{\Omega=U \backslash \bar{\omega} ; \omega \text { open set, } \omega \subset V, \# \bar{\omega} \leq k\} .
$$

For any $\Omega \in \mathcal{O}_{k}, k \geq 1$, the boundary of $\Omega$ can be split into $\partial \Omega=\Gamma_{N} \cup \partial U$ with $\Gamma_{N}=\partial \omega$. The boundary $\partial U$ is divided in two components $\partial U=\Gamma_{S} \cup \Gamma_{D}$. The boundaries $\Gamma_{N}$ and $\Gamma_{D}$ will have Neumann and Dirichlet boundary conditions, respectively, whereas Signorini conditions will be imposed on $\Gamma_{S}$. Let us point out that the open set $\omega$ is not necessarily any connected set as illustrated in Fig. 1.

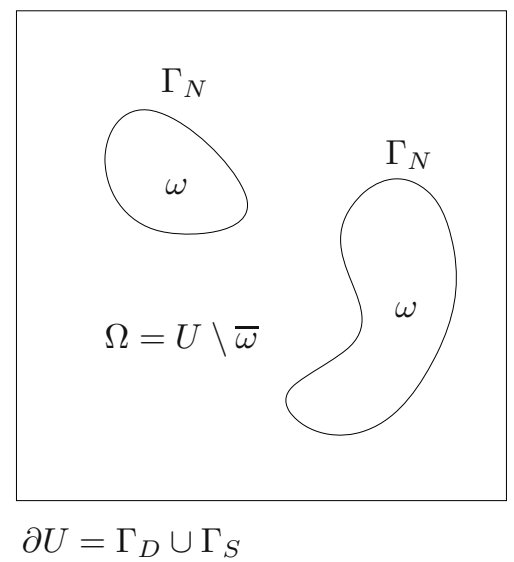

Fig. 1. Admissible domain $\Omega$.

For $f \in C^{\infty}(\bar{U})$, we consider the following Signorini problem:

$$
\left\{\begin{aligned}
-\Delta u+u & =f \text { in } \quad \Omega, \\
u & =0 \text { on } \Gamma_{D}, \\
\partial_{n} u & =0 \text { on } \Gamma_{N}, \\
u \geq 0, \partial_{n} u \geq 0, u \partial_{n} u=0 & \text { on } \Gamma_{S},
\end{aligned}\right.
$$

where $n$ is the unit outward normal vector to $\partial \Omega$ and $\partial_{n}$ stands for the normal derivative on $\partial \Omega$. The Signorini problem (2) admits a unique weak solution $u(\Omega) \in K(\Omega)$ satisfying the variational inequality

$$
\begin{array}{r}
\int_{\Omega} \nabla u \cdot \nabla(v-u) \mathrm{d} x \geq \int_{\Omega}(f-u)(v-u) \mathrm{d} x, \\
\forall v \in K(\Omega),
\end{array}
$$

with

$$
K(\Omega)=\left\{v \in H_{\Gamma_{D}}^{1}(\Omega) \mid v \geq 0 \text { a.e. on } \Gamma_{S}\right\},
$$

and where $H_{\Gamma_{D}}^{1}(\Omega)$ stands for the classical Sobolev space of functions which belong to $H^{1}(\Omega)$ and with null traces on the boundary $\Gamma_{D}$.

Now, let us consider the energy functional

$$
E(\Omega, u)=\frac{1}{2} \int_{\Omega}\left(|\nabla u|^{2}+u^{2}\right) \mathrm{d} x-\int_{\Omega} f u \mathrm{~d} x .
$$

Observe that the energy $E(\Omega, u)$ can also be written as

$$
\begin{aligned}
E(\Omega, u) & =-\frac{1}{2} \int_{\Omega}\left(|\nabla u|^{2}+u^{2}\right) \mathrm{d} x \\
& =-\frac{1}{2} \int_{\Omega} f u \mathrm{~d} x .
\end{aligned}
$$

In this paper, we are interested in the shape functional

$$
J(\Omega)=E(\Omega, u)+\lambda A(\Omega)-\mu P_{c}(\Omega)^{2},
$$

where $A(\Omega)$ and $P_{c}(\Omega)$ are defined by

$$
\begin{aligned}
A(\Omega) & =|\Omega|, \\
P_{c}(\Omega) & =\max (0, \ell(\partial \Omega)-c) .
\end{aligned}
$$

In the above definitions, $|\Omega|$ denotes the Lebesgue measure of $\Omega$ in $\mathbb{R}^{2}$ and $\ell(\partial \Omega)$ is the 1-dimensional Hausdorff measure of $\partial \Omega$. The constants $\lambda$ and $\mu$ are positive and allow us to take into account the area and the perimeter constraint, respectively. The constant $c$ is positive and defines a shift of the perimeter beyond which the perimeter constraint becomes active. All constants have to be well chosen for efficient and appropriate solutions of the numerical problem.

For any $k \geq 1$, we are interested in the following shape optimization problem:

$$
\max \left\{J(\Omega): \Omega \in \mathcal{O}_{k}\right\}
$$

Since the exterior boundary of $\Omega$ is fixed to be $\partial U$, the domains $\Omega$ admissible in (10) are actually determined by their internal boundary $\Gamma_{N}$. We refer the reader to (Laurain, 2006) for an existence result of an optimal domain in (10) for the case of the linear problem, i.e., with $\Gamma_{S}=\emptyset$.

In the following two sections, we study the shape differentiability, and we perform an asymptotic analysis which results in topological derivatives of the functional $J$. To make the paper self-contained, we provide the proofs in Appendix. Then, in Section 5, we construct a level set representation based on the shape derivative of $J$. This formulation provides a practical way to increase the numerical values of the shape functional $J$. The appearance of a new hole is not possible with the only use of the level set method based on the shape derivatives. In particular, the knowledge of topological derivatives allows us to create a new hole in order to increase $J$.

Finally, in Section 6, a numerical algorithm of shape optimization is described. The finite element method for the Signorini problem is introduced in Section 7. In Section 8, a finite difference method for Hamilton-Jacobi equations is introduced. Numerical results are presented in Section 9. 


\section{Shape Derivative}

The shape derivatives of solutions for the Signorini problem can be evaluated by an application of the abstract result on Hadamard differentiability of the projection operator onto convex sets in Hilbert spaces (Jarusek et al., 2003). A complete study of shape differentiation of solutions of elliptic equations and variational inequalities can be found in the monographs (Delfour and Zolesio, 2001; Henrot and Pierre, 2005; Sokołowski and Zolesio, 1992)

Let $\delta \geq 0$ be a given parameter and $\xi \in C_{0}^{\infty}(U)$ a given vector field. We consider the mapping $F_{\delta}=I+\delta \xi$ and we set $\Omega_{\delta}=F_{\delta}(\Omega)$. Since $\xi$ has compact support in $U$, for small $\delta$ we have that $\Omega_{\delta} \subset U$ and $F_{\delta}(\partial U)=\partial U$, that is, the exterior boundary of $\Omega$ is maintained fixed.

There exists a unique element $u_{\delta} \in K_{\delta}\left(\Omega_{\delta}\right)$ given by a solution to the following variational inequality: Find $u_{\delta} \in K_{\delta}\left(\Omega_{\delta}\right)$ such that for all $v \in K_{\delta}\left(\Omega_{\delta}\right)$

$$
\int_{\Omega_{\delta}} \nabla u_{\delta} \cdot \nabla\left(v-u_{\delta}\right) \mathrm{d} x \geq \int_{\Omega_{\delta}}\left(f-u_{\delta}\right)\left(v-u_{\delta}\right) \mathrm{d} x,
$$

where

$$
\begin{aligned}
K_{\delta}\left(\Omega_{\delta}\right)=\left\{v \in H^{1}\left(\Omega_{\delta}\right) \mid\right. & v=0 \text { a.e. on } \Gamma_{D}, \\
& \left.v \geq 0 \text { a.e. on } \Gamma_{S}\right\} .
\end{aligned}
$$

We assume that $\Omega$ is a smooth domain. It can be easily shown that the limit

$$
\mathrm{d} J(\Omega, \xi):=\lim _{\delta \rightarrow 0} \frac{J\left(\Omega_{\delta}\right)-J(\Omega)}{\delta}
$$

exists and is equal to

$$
\begin{aligned}
\mathrm{d} J(\Omega ; \xi)= & \int_{\Gamma_{N}}\left(\frac{1}{2}|\nabla u|^{2}+\frac{1}{2} u^{2}-f u\right)\langle\xi, n\rangle \mathrm{d} \sigma \\
& +\int_{\Gamma_{N}}\left(\lambda-2 \mu P_{c}(\Omega) \mathcal{H}\right)\langle\xi, n\rangle \mathrm{d} \sigma,
\end{aligned}
$$

where $\mathcal{H}$ is the curvature of the boundary $\Gamma_{N}$ and $n$ is the unit normal vector to $\Gamma_{N}$, directed outwards of $\Omega$.

\section{Topological Derivative}

4.1. Topological Derivatives for Variational Inequalities. A method for the evaluation of topological derivatives for variational inequalities is proposed in (Sokołowski and Żochowski, 2005a). The method is based on the so-called conical differentiability of solutions to variational inequalities with respect to the coefficients of the governing differential operator. It is required that the metric projection in the energy space onto the convex set $K$ be Hadamard differentiable with respect to the perturbations of the point. Such a property is sufficient to obtain the directional differentiability of solutions to the variational inequality with respect to the boundary variations and, as a result, with respect to the changes in the topology by the creation of a small hole. We derive the result for a specific problem, and obtain the topological derivative of the energy functional, which is, in fact, of the same form as in the case of a linear problem. However, the proof of this result is not the same and from the technical point of view it is much more involved. In particular, the results obtained by the linearization of nonlinear problems are not applicable to the Signorini problem, which cannot be linearized. Therefore, the so-called truncation method (Amstutz and Andrä, 2006) cannot be used in order to obtain the topological derivatives for variational inequalities. The reason is that in the case of variational inequalities the adjoint state cannot be introduced.

\subsection{Topological Derivative of the Energy Functional.} For simplicity, in this section we assume that $\Omega=U$ (i.e., $\Gamma_{N}=\emptyset$ ) and that the boundary $\partial U$ will only receive the Signorini conditions (i.e., $\Gamma_{D}=\emptyset$ ), so that $\partial \Omega=\partial U=\Gamma_{S}$. Let us now consider the perforated domain $\Omega_{\rho}=U \backslash \bar{B}_{\rho}$, where $B_{\rho}$ is the ball of radius $\rho$, centered at $x_{0}$ and with boundary $\Gamma_{\rho}=\partial B_{\rho}$. In order to study the topological derivative of the functional $J$ for the nonlinear Signorini problem, we need to derive the asymptotic expansion with respect to $\rho$ of the energy $E\left(\Omega_{\rho}, u_{\rho}\right)$, where $u_{\rho}$ is the solution to the Signorini problem

$$
\left\{\begin{aligned}
-\Delta u_{\rho}+u_{\rho}=f & & \text { in } \Omega_{\rho}, \\
u_{\rho} \geq 0, \partial_{n} u_{\rho} \geq 0, u_{\rho} \partial_{n} u_{\rho}=0 & & \text { on } \Gamma_{S}, \\
\partial_{n} u_{\rho}=0 & & \text { on } \Gamma_{\rho} .
\end{aligned}\right.
$$

To this end, we shall make use of the so-called truncated domain technique (Masmoudi, 2002; Sokołowski and Żochowski, 2005a). In this section we explain the main lines of the technique used in order to derive the asymptotic expansion of the functional $J$. The detailed proofs of the results of this section can be found in Appendix.

Now, we describe the truncated domain technique for the Signorini problem. Let us denote by $\Omega_{R}$ the domain

$$
\Omega_{R}=U \backslash \bar{B}_{R},
$$

where $B_{R}$ is the ball of radius $R$ with $R>\rho$, centered at $x_{0}$, and we define the ring $C(R, \rho)$ such that $\Omega_{\rho}=$ $\Omega_{R} \cup \Gamma_{R} \cup C(R, \rho)$. We consider the following truncated problem:

$$
\left\{\begin{array}{r}
-\Delta u_{\rho}^{R}+u_{\rho}^{R}=f \text { in } \Omega_{R}, \\
u_{\rho}^{R} \geq 0, \partial_{n} u_{\rho}^{R} \geq 0, u_{\rho}^{R} \partial_{n} u_{\rho}^{R}=0 \text { on } \Gamma_{S}, \\
-\partial_{n} y_{\rho}+\partial_{n} u_{\rho}^{R}=A_{\rho}\left(u_{\rho}^{R}\right) \text { on } \Gamma_{R} .
\end{array}\right.
$$


In the above problem, $A_{\rho}$ is the Steklov-Poincaré operator defined by

$$
A_{\rho}:\left\{\begin{array}{rlc}
H^{\frac{1}{2}}\left(\Gamma_{R}\right) & \rightarrow & H^{-\frac{1}{2}}\left(\Gamma_{R}\right) \\
v & \mapsto & \partial_{n} w_{\rho}
\end{array},\right.
$$

where $w_{\rho}=w_{\rho}(v)$ is the unique solution of the problem

$$
\left\{\begin{aligned}
-\Delta w_{\rho}+w_{\rho}=0 & \text { in } C(R, \rho), \\
w_{\rho}=v & \text { on } \Gamma_{R}, \\
\partial_{n} w_{\rho}=0 & \text { on } \Gamma_{\rho},
\end{aligned}\right.
$$

with a given $v \in H^{\frac{1}{2}}\left(\Gamma_{R}\right)$.

Finally, the function $y_{\rho}$ appearing in the problem (17) is the solution to the following problem:

$$
\left\{\begin{aligned}
-\Delta y_{\rho}+y_{\rho} & =f_{\mid C(R, \rho)} & & \text { in } C(R, \rho), \\
y_{\rho} & =0 & & \text { on } \Gamma_{R}, \\
\partial_{n} y_{\rho} & =0 & & \text { on } \Gamma_{\rho} .
\end{aligned}\right.
$$

Then the following result can be easily proved.

Proposition 1. The solution $u_{\rho}^{R}$ to the problem (17) satisfies

$$
u_{\rho}^{R}=u_{\rho \mid \Omega_{R}},
$$

and we also have

$$
u_{\rho \mid C(R, \rho)}=w_{\rho}\left(u_{\rho}^{R}\right)+y_{\rho},
$$

with $w_{\rho}$ and $y_{\rho}$ denoting the solutions to (19) and (20), respectively.

In order to obtain the topological derivative of $J$, we have to perform an asymptotic analysis of the energy functional $E\left(\Omega_{\rho}, u_{\rho}\right)$ with respect to the small radius $\rho$. Recall that, cf. (6),

$$
E\left(\Omega_{\rho}, u_{\rho}\right)=-\frac{1}{2} \int_{\Omega_{\rho}}\left(\left|\nabla u_{\rho}\right|^{2}+u_{\rho}^{2}\right) \mathrm{d} x .
$$

Using the domain truncation technique, in the version adapted to our problem (Sokołowski and Żochowski, 2005a), we can split the integral in two parts and obtain

$$
E\left(\Omega_{\rho}, u_{\rho}\right)=E\left(\Omega_{R}, u_{\rho}^{R}\right)-\frac{1}{2} E_{\rho}^{(1)}\left(u_{\rho}^{R}\right)+\frac{1}{2} E_{\rho}^{(2)}(f),
$$

where

$$
E\left(\Omega_{R}, u_{\rho}^{R}\right)=-\frac{1}{2} \int_{\Omega_{R}}\left(\left|\nabla u_{\rho}^{R}\right|^{2}+\left(u_{\rho}^{R}\right)^{2}\right) \mathrm{d} x
$$

and

$$
E_{\rho}^{(1)}\left(u_{\rho}^{R}\right)=\int_{C(R, \rho)}\left(\left|\nabla w_{\rho}\right|^{2}+w_{\rho}^{2}\right) \mathrm{d} x,
$$

with $w_{\rho}=w_{\rho}\left(u_{\rho}^{R}\right)$ and

$$
E_{\rho}^{(2)}(f)=-\int_{C(R, \rho)}\left(\left|\nabla y_{\rho}\right|^{2}+y_{\rho}^{2}\right) \mathrm{d} x .
$$

Using an abstract result on the conical differentiability of the solution of a variational inequality given in (Jarusek et al., 2003), we can adapt a result from (Sokołowski and Żochowski, 2005a) and show that the solution $u_{\rho}^{R}$ of (2) on the truncated domain $\Omega_{R}$ admits the following expansion:

$$
u_{\rho}^{R}-u_{0}^{R}=O\left(\rho^{2}\right) .
$$

Actually, it can be proved that there exists a function $q$ called the exterior topological derivative of the solution $u$ to the Signorini problem (2) such that

$$
u_{\rho}^{R}=u_{0}^{R}+\rho^{2} q+o\left(\rho^{2}\right) .
$$

This function $q$ is the unique solution of a variational inequality and does not depend on $\rho$. More precisely, the following result holds true (see (Rao and Sokołowski, 2000) for a simple proof of the conical differentiability for the Signorini problem):

Proposition 2. The exterior topological derivative is given by the restriction of the solution to the problem

$$
q \in \mathcal{S}_{K}(u): a(q, \varphi-q)+b(u, \varphi-q) \geq 0
$$

to $\Omega_{R}$ for all $\varphi \in \mathcal{S}_{K}(u)$. In the above, the bilinear form $a$ is given by $a(u, v)=\int_{\Omega} \nabla u \cdot \nabla v+u v$, the bilinear form $b(u, v)$ is the first variation of the energy functional (see (Sokotowski and Żochowski, 2003) for an equivalent expression of such a variation), and the cone is defined by

$$
\begin{gathered}
\mathcal{S}_{K}(u)=\left\{v \in H_{\Gamma_{D}}^{1}(\Omega) \mid v \geq 0 \text { a.e. on } \Xi(u),\right. \\
a(u, v)=(f, v)\}
\end{gathered}
$$

with the coincidence set $\Xi(u)=\left\{x \in \Gamma_{S} \mid u(x)=0\right\}$. Moreover, the expansion (28) of $u_{\rho}^{R}$ with respect to $\rho$ is valid in $\Omega_{R}$, for all $R>0$.

The expansion (27) allows us to perform the asymptotic expansion of (23) and we obtain (see the Appendix for the proof)

$$
\begin{aligned}
& E\left(\Omega_{\rho}, u_{\rho}\right) \\
& =E(\Omega, u)-\left[\frac{u\left(x_{0}\right)^{2}}{2}+\left|\nabla u\left(x_{0}\right)\right|^{2}-f\left(x_{0}\right) u\left(x_{0}\right)\right] \\
& \cdot \pi \rho^{2}+o\left(\rho^{2}\right) .
\end{aligned}
$$

Now, using the expansions

$$
\begin{aligned}
A\left(\Omega_{\rho}\right) & =A(\Omega)-\pi \rho^{2}, \\
P_{c}\left(\Omega_{\rho}\right)^{2} & =P_{c}(\Omega)^{2}+4 \pi P_{c}(\Omega) \rho+o\left(\rho^{2}\right),
\end{aligned}
$$


we obtain the asymptotic expansion for $J$.

Theorem 1. We have the following expansion of $J\left(\Omega_{\rho}\right)$ :

$$
\begin{aligned}
& J\left(\Omega_{\rho}\right) \\
& \quad=J(\Omega) \\
& \quad-\left[\frac{u\left(x_{0}\right)^{2}}{2}+\left|\nabla u\left(x_{0}\right)\right|^{2}-f\left(x_{0}\right) u\left(x_{0}\right)+\lambda\right] \pi \rho^{2} \\
& \quad-4 \mu \pi P_{c}(\Omega) \rho+o\left(\rho^{2}\right)
\end{aligned}
$$

and the topological derivative $\mathcal{T}_{\Omega}\left(x_{0}\right)$ of the functional $J$ at point $x_{0} \in \Omega$ is given by

$$
\mathcal{T}_{\Omega}\left(x_{0}\right)= \begin{cases}-\left|\nabla u\left(x_{0}\right)\right|^{2}-\frac{1}{2} u\left(x_{0}\right)^{2}+u f\left(x_{0}\right)-\lambda \\ & \text { if } P_{c}(\Omega)=0, \\ -4 \mu P_{c}(\Omega) & \text { if } P_{c}(\Omega)>0 .\end{cases}
$$

\section{Level Set Formulation}

5.1. Hamilton-Jacobi Equation. The basic idea of the level set method is to represent a domain and its boundary as level sets of a continuous function $\phi$ defined on the whole domain $U$.

Let us consider the evolution of a domain $\Omega \subset U \subset$ $\mathbb{R}^{2}$ under a velocity field $\xi$. More precisely, we define $\Omega_{t}=(I+t \xi)(\Omega), t \in \mathbb{R}^{+}$, with a smooth vector field $\xi$. The domain and the boundary are defined by a function $\phi=\phi(x, t)$ such that

$$
\Omega_{t}=\{x \in U, \phi(x, t)<0\}
$$

and

$$
\partial \Omega_{t}=\{x \in U, \phi(x, t)=0\},
$$

i.e., the boundary $\partial \Omega_{t}$ is the level curve of the function $\phi$ (see Fig. 2).

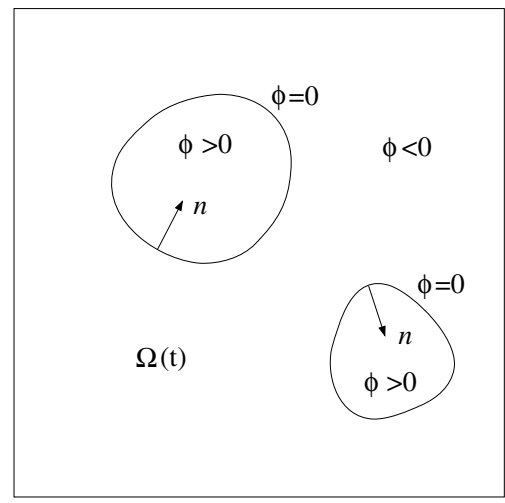

Fig. 2. Domain and level set function.

Let $x(t)$ be the position of a particle on the boundary $\partial \Omega_{t}$ moving with velocity $\xi=\dot{x}(t)$. Differentiating the relation $\phi(x(t), t)=0$ with respect to $t$ leads to the transport equation

$$
\phi_{t}+\xi \cdot \nabla \phi=0
$$

Moreover, the normal directions $n$ to the level sets of $\phi$ are given by $n=\nabla \phi /|\nabla \phi|$. The evolution of $\phi$ is then governed by the Hamilton-Jacobi equation

$$
\phi_{t}+\xi_{n}|\nabla \phi|=0 \quad \text { in } U \times \mathbb{R}^{+},
$$

where $\xi_{n}$ is the normal velocity (the normal component of $V$ ), i.e., $\xi_{n}=\langle\xi, n\rangle$. Initial data and boundary conditions have to be imposed together with the HamiltonJacobi equation (37). The initial data $\phi(0, x)=\phi_{0}(x)$ are chosen as the signed distance function to the initial boundary $\partial \Omega_{0}=\partial \Omega$, i.e.,

$$
\phi_{0}(x)= \pm \operatorname{dist}\left(x, \partial \Omega_{0}\right),
$$

with the minus (resp. plus) sign if the point $x$ is inside (resp. outside) the initial domain $\Omega_{0}=\Omega$.

A boundary condition also has to be imposed on the part of the boundary $\partial U$ of the domain $U$ where the normal velocity $\xi_{n}$ is negative, i.e., where the velocity is directed inwards the domain $U$. Alternatively, we decide to impose a homogeneous Neumann boundary condition on the whole boundary $\partial U$ :

$$
\partial_{n} \phi=0 \text { on } \partial U \text {. }
$$

5.2. Normal Velocity for the Level Set Equation. When a hole is created inside the domain, the boundary conditions for the state equation on the boundary of the hole are of the Neumann type. The shape derivative is then given by (14). Since we locally (i.e., under small perturbations of the domain) require that the condition $\mathrm{d} J(\Omega ; \xi)>0$ be satisfied, this leads to the following choice for the normal component $\xi_{n}=\langle\xi, n\rangle$ of the velocity field $\xi$ :

$$
\xi_{n}=\frac{1}{2}|\nabla u|^{2}+\frac{1}{2} u^{2}-u f+\lambda-2 \mu P_{c}(\Omega) \mathcal{H} \text { on } \Gamma_{N} .
$$

With a velocity field $\xi$ satisfying (40), we clearly have $\mathrm{d} J(\Omega ; \xi)>0$ and then $J\left(\Omega_{t}\right)>J(\Omega)$ for $t$ small enough.

The Hamilton-Jacobi equation (37) necessitates velocity fields defined in the whole domain $U$. So, we need an extension to $U$ of the normal velocity, given by the shape gradients in the following form:

$$
\xi_{n}=V_{n}-2 \mu P_{c}(\Omega) \mathcal{H} \text { on } \Gamma_{N},
$$

with

$$
V_{n}=\frac{1}{2}|\nabla u|^{2}+\frac{1}{2} u^{2}-u f+\lambda \text { on } \Gamma_{N} .
$$


The curvature $\mathcal{H}$ is given by

$$
\mathcal{H}=\operatorname{div}\left(\frac{\nabla \phi}{|\nabla \phi|}\right)
$$

and, therefore, $\mathcal{H}$ is defined in $U$. The normal velocity part $V_{n}$ given by (42) has to be extended outside the boundary $\Gamma_{N}$. Let $V_{\text {ext }}$ denote such an extension (see Section 8.1 below for a detailed construction). The Hamilton-Jacobi equation is then

$$
\phi_{t}+\left(V_{\text {ext }}-2 \mu P_{c}(\Omega) \operatorname{div}\left(\frac{\nabla \phi}{|\nabla \phi|}\right)\right)|\nabla \phi|=0 \text { in } U \times \mathbb{R}^{+} \text {. }
$$

\section{Shape Optimization Algorithm}

Let us now describe the steps of the general shape optimization algorithm.

\section{First step: Initial domain}

First of all, we choose an initial domain $\Omega^{0}$ and we compute the solution to the Signorini problem (2) in $\Omega^{0}$. This is performed using a piecewise linear finite element method on appropriate (unstructured) meshes with the Uzawa algorithm for the treatment of the boundary constraint (see Section 7 for details). Then we compute the topological derivative $\mathcal{T}_{\Omega^{0}}(x)$ for all $x \in \Omega^{0}$, according to (33).

\section{Second step: Creating a hole}

We use the topological derivative to create a hole in the domain $\Omega^{0}$. More precisely, we find a point $x_{0} \in \Omega^{0}$ such that $\mathcal{T}_{\Omega^{0}}\left(x_{0}\right)=\max _{x \in \Omega^{0}} \mathcal{T}_{\Omega^{0}}(x)$. If $\mathcal{T}_{\Omega^{0}}\left(x_{0}\right)>0$, then we create a circular hole $\omega_{\rho}$ of a small radius $\rho>0$, centered at $x_{0}$. We denote by $\Omega_{*}^{0}$ the new domain with the hole. A Neumann condition will be imposed on the boundary of the new hole. Observe that the radius of this hole should be as small as possible, depending on the space step of the mesh.

\section{Third step: Evolution}

Now we proceed with the evolution of the domain $\Omega_{*}^{0}$. We need to compute the solution $\phi$ to the Hamilton-Jacobi equation (37)-(39) with (41)-(43). The initial $\phi$ is taken as the signed distance function on the domain $\Omega_{*}^{0}$. According to (40), we compute the normal velocity $\xi_{n}$ on the internal boundary part $\Gamma_{N}$ of $\partial \Omega_{*}^{0}$. Remark that this requires a new computation of the solution for the Signorini problem in $\Omega_{*}^{0}$. Since the normal velocity $\xi_{n}$ is only known on the boundary part $\Gamma_{N}$, we need to extend $\xi_{n}$ to the whole domain $U$. This is required in order to solve the level set equation (37) in $U$. The next section will explain how to proceed with the construction of the extended normal velocity in a numerically accurate way.

Several iterations of the Hamilton-Jacobi equations are needed in order to determine the new domain $\Omega^{1}$. Then we go back to the first step with $\Omega^{1}$, which replaces $\Omega^{0}$.

\section{Numerical Method for the Signorini Problem}

We use a piecewise linear finite element method with the Uzawa algorithm to compute a solution to the Signorini problem (2). The Uzawa algorithm is used for the nonnegative boundary constraint on $\Gamma_{S}$. In the finite element framework, we are looking for $u_{h} \in \mathcal{U} \subset \mathbb{R}^{n}$ such that

$$
E_{h}\left(u_{h}\right)=\inf _{v \in \mathcal{U}} E_{h}(v)
$$

where $\mathcal{U}=\left\{v \in \mathbb{R}^{n} \mid C v \geq 0\right\}$ and where the approximate energy functional

$$
E_{h}(v)=\frac{1}{2}(A v, v)-(b, v)
$$

corresponds to the finite element discretization of the energy functional

$$
E(v)=\frac{1}{2}\left(\int_{\Omega}|\nabla v|^{2}+v^{2} \mathrm{~d} x\right)-\int_{\Omega} f v \mathrm{~d} x .
$$

In the above definition of $E_{h}$, the matrix $A \in \mathcal{M}_{n \times n}(\mathbb{R})$ is the usual stiffness-mass matrix associated with natural Neumann boundary conditions on $\Gamma_{S}$. The vector $b \in \mathbb{R}^{n}$ is given by the finite element discretization of the source term $f$. The matrix $C \in \mathcal{M}_{m \times n}(\mathbb{R})$ in the definition of the space $\mathcal{U}$ selects the entries of a vector only on the nodes located at the boundary $\Gamma_{S}$ ( $m$ is the number of nodes on the Signorini boundary $\Gamma_{S}$ ).

The Uzawa algorithm consists in the computation of a sequence $\left(u_{h}^{k}, \lambda_{h}^{k}\right) \in \mathbb{R}^{n} \times \mathbb{R}^{+}, k \geq 0$, defined by the following relations:

- $A u_{h}^{k}-b-C^{T} \lambda_{h}^{k}=0$,

- $\lambda_{h}^{k+1}=\max \left(\lambda_{h}^{k}-\rho\left(C u_{h}^{k}\right)_{i}, 0\right)$ for $1 \leq i \leq m$.

Under the condition that $0<\rho<2 \lambda_{1}(A) /\|C\|^{2}$, where $\lambda_{1}(A)$ denotes the smallest eigenvalue of $A$, the sequence $u_{h}^{k}$ converges to a finite element approximation $u_{h}$ of the Signorini problem (2).

\section{Numerical Method for the Level Set Equation}

Now we describe how to construct the extended normal velocity on the whole domain $U$ and how to solve the level set equation (37).

Let us start with a general remark on the numerical solution of (37). For the sake of numerical accuracy, the solution of the level set equation (37) should be neither too flat nor too steep. This condition is fulfilled, e.g., if $\phi$ is the distance function, i.e., $|\nabla \phi|=1$. Unfortunately, even if we start with a (signed) distance function for the initial data $\phi_{0}$, the solution $\phi$ of the level set equation (37) does 
not generally remain close to any distance function. We can perform a reinitialization of $\phi$ at a time $t$ by determining the solution $\varphi=\varphi(x, \tau)$ of the following equation, up to the stationary state (Peng et al., 1999):

$$
\begin{aligned}
\varphi_{\tau}+S(\phi)(|\nabla \varphi|-1) & =0 \text { in } U \times \mathbb{R}^{+}, \\
\varphi(x, 0) & =\phi(x, t), \quad x \in U,
\end{aligned}
$$

Here $S$ is an approximation to the sign function, i.e.,

$$
S(d)=\frac{d}{\sqrt{d^{2}+|\nabla d|^{2} \varepsilon^{2}}}
$$

with $\varepsilon=\min (\Delta x, \Delta y)$, where $\Delta x$ and $\Delta y$ stand for the space discretization steps in the $x$ and $y$ directions, respectively (see below). Other choices are also possible for the approximate sign function. We refer the reader to (Peng et al., 1999) for details.

8.1. Extended Normal Velocity. The normal velocity part $V_{n}$ (see (42)) should be defined on the whole domain $U$ for the well posedness of the level set equation (37). Since the normal velocity $V_{n}$ is only given on the boundary $\Gamma_{N}$, we need to extend $V_{n}$ to the domain $U$. Another reason for extending the velocity is to enforce the solution $\phi$ of the level set equation to remain (close to) the distance function. Indeed, if we are able to compute an extended normal velocity $V_{\text {ext }}$ such that

$$
\nabla V_{\text {ext }} \cdot \nabla \phi=0 \text { in } U \times \mathbb{R}^{+},
$$

then it can be shown (Zhao et al., 1996) that the solution $\phi$ to the level set equation (37) satisfies $|\nabla \phi|=1$. A way to construct an extension $V_{\text {ext }}$ satisfying (48) at time $t$ is to solve the following equation for $q$, up to the stationary state (Osher and Fedkiw, 2004; Peng et al., 1999):

$$
\begin{aligned}
q_{\tau}+S(\phi) \frac{\nabla \phi}{|\nabla \phi|} \cdot \nabla q & =0 \quad \text { in } \quad U \times \mathbb{R}^{+} \\
q(x, 0) & =p(x, t), \quad x \in U,
\end{aligned}
$$

where $p$ equals $V_{n}$ given by (42) on the boundary $\Gamma_{N}$ and 0 elsewhere. The function $S$ is the approximate sign function defined by (47).

8.2. Discretization of the Level Set Equation. We fix $U$ as the unit square $U=(0,1) \times(0,1)$. For the discretization of the Hamilton-Jacobi equation (37), we first define the mesh grid of $U$. We introduce the nodes $P_{i j}$ whose coordinates are given by $(i \Delta x, j \Delta y)$, where $\Delta x$ and $\Delta y$ are the discretization steps in the $x$ and $y$ directions, respectively. Let us also denote by $t^{k}=k \Delta t$ the discrete time for $k \in \mathbb{N}$, where $\Delta t$ is the time step. We are seeking an approximation $\phi_{i j}^{k} \simeq \phi\left(P_{i j}, t^{k}\right)$. The numerical scheme we use was proposed in (Osher and Fedkiw, 2004; Osher and Sethian, 1988; Sethian, 1996). This explicit upwind scheme is

$$
\phi_{i j}^{k+1}=\phi_{i j}^{k}-\Delta \operatorname{tg}\left(D_{-}^{x} \phi_{i j}^{k}, D_{+}^{x} \phi_{i j}^{k}, D_{-}^{y} \phi_{i j}^{k}, D_{+}^{y} \phi_{i j}^{k}\right),
$$

where

$$
D_{-}^{x} \phi_{i j}=\frac{\phi_{i j}-\phi_{i-1, j}}{\Delta x}, \quad D_{+}^{x} \phi_{i j}=\frac{\phi_{i+1, j}-\phi_{i j}}{\Delta x}
$$

are respectively the backward and forward approximations of the $x$-derivative of $\phi$ at $P_{i j}$. Similar expressions hold for the approximations $D_{-}^{y}$ and $D_{+}^{y}$ of the $y$ derivative. The numerical flux is given by

$$
g\left(D_{-}^{x} \phi_{i j}, D_{+}^{x} \phi_{i j}, D_{-}^{y} \phi_{i j}, D_{+}^{y} \phi_{i j}\right) g_{i j}^{(1)}+g_{i j}^{(2)} .
$$

The numerical flux part $g_{i j}^{(1)}$ corresponds to the discretization of the first-order part of the Hamilton-Jacobi equation and is given by

$$
g_{i j}^{(1)}=\max \left(v_{i j}, 0\right) G^{+}+\min \left(v_{i j}, 0\right) G^{-}
$$

with

$$
\begin{aligned}
G^{+}= & {\left[\max \left(D_{-}^{x} \phi_{i j}, 0\right)^{2}+\min \left(D_{+}^{x} \phi_{i j}, 0\right)^{2}\right.} \\
& \left.+\max \left(D_{-}^{y} \phi_{i j}, 0\right)^{2}+\min \left(D_{+}^{y} \phi_{i j}, 0\right)^{2}\right]^{1 / 2}, \\
G^{-}= & {\left[\min \left(D_{-}^{x} \phi_{i j}, 0\right)^{2}+\max \left(D_{+}^{x} \phi_{i j}, 0\right)^{2}\right.} \\
& \left.+\min \left(D_{-}^{y} \phi_{i j}, 0\right)^{2}+\max \left(D_{+}^{y} \phi_{i j}, 0\right)^{2}\right]^{1 / 2},
\end{aligned}
$$

and $v_{i j}=V_{\text {ext }}\left(P_{i j}\right)$ is the extended normal velocity at point $P_{i j}$.

The numerical flux part $g_{i j}^{(2)}$ in (53) is the centered finite difference approximation of the second-order term of the Hamilton-Jacobi equation, i.e., $g_{i j}^{(2)} \simeq$ $-2 \mu P_{c}(\Omega) \mathcal{H}|\nabla \phi|\left(P_{i j}\right)$.

This upwind scheme is stable under the following two conditions:

$$
\begin{gathered}
\left(\max _{U}\left|V_{\text {ext }}\right|\right) \frac{\Delta t}{\min (\Delta x, \Delta y)} \leq \frac{1}{2 \sqrt{2}} \\
4 \mu P_{c}(\Omega) \frac{\Delta t}{\min \left(\Delta x^{2}, \Delta y^{2}\right)} \leq 1
\end{gathered}
$$

The condition (54) arises from the upwind part of the scheme corresponding to the discretization of the firstorder term of the Hamilton-Jacobi equation. The condition (55) comes from the centered difference discretization used for the second-order term involving the curvature. 
8.3. Computing the Extended Velocity. At each iteration $k$ of the previous scheme, we compute the extended normal velocity $V_{\text {ext }}$ as the stationary solution of (49) and (50). We compute $q_{i j}^{n} \simeq q\left(P_{i j}, t^{n}\right)$ from the following upwind approximation of (49) :

$$
\begin{aligned}
q_{i j}^{n+1}= & q_{i j}^{n}-\Delta \tau\left[\max \left(s_{i j} n_{i j}^{x}, 0\right) D_{-}^{x} q_{i j}\right. \\
& +\min \left(s_{i j} n_{i j}^{x}, 0\right) D_{+}^{x} q_{i j} \\
& +\max \left(s_{i j} n_{i j}^{y}, 0\right) D_{-}^{y} q_{i j} \\
& \left.+\min \left(s_{i j} n_{i j}^{y}, 0\right) D_{+}^{y} q_{i j}\right],
\end{aligned}
$$

where $s_{i j}=S\left(\phi_{i j}^{n}\right)$. We use central differences to compute the approximation $n_{i j}$ of the unit normal vector $n=$ $\left(n^{x}, n^{y}\right)=\left(\phi_{x} / \sqrt{\phi_{x}^{2}+\phi_{y}^{2}}, \phi_{y} / \sqrt{\phi_{x}^{2}+\phi_{y}^{2}}\right)$ at node $P_{i j}$. The initial value $q_{0}$ coincides with $V_{n}$ at the grid points with the distances to the interface less than $\min (\Delta x, \Delta y)$ and is zero elsewhere.

\section{Numerical Results}

We fix $U$ as the unit square $U=(0,1) \times(0,1)$ and we choose $\Gamma_{D}=\emptyset$, i.e., Signorini conditions are imposed on the external boundary $\partial U$.

First numerical example. We first present a numerical computation performed with

$$
\lambda=0.3, \quad \mu=0.001, \quad c=4.6 .
$$

The source term $f$ is chosen with compact support in $U$ and is given by (see Fig. 3)

$$
f= \begin{cases}10 & \text { in }[0.2,0.4]^{2} \\ -10 & \text { in }[0.6,0.8]^{2} \\ 0 & \text { elsewhere }\end{cases}
$$

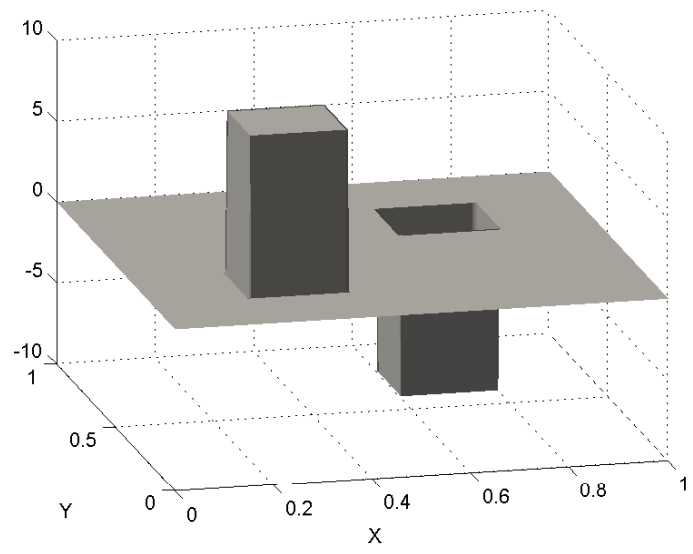

Fig. 3. First numerical example: the source function $f$.
The initial domain is chosen as $U \backslash \bar{\omega}_{0}$, where $\omega_{0}$ is a circle of radius 0.25 centered at $(0.5,0.5)$ (see the first plot of Fig. 5). We observe that the initial hole $\omega_{0}$ disappears thanks to the use of the shape derivative in the level set formulation. On the other hand, two other holes are created in different locations by the application of the topological derivative. We also observe that the functional $J$ converges to a maximum which is equal to 0.2798122 , while the domain also converges to an optimal domain $\Omega$ (see Fig. 5). The numerical solution $u$ and its gradient are represented in Fig. 4 when the optimal domain is achieved.

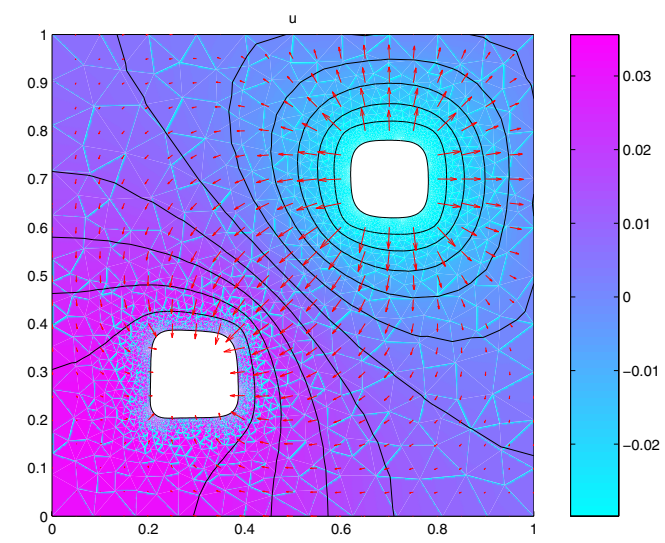

Fig. 4. First numerical example: the numerical solution $u$ on the optimal domain.

Second numerical example. Now we present a numerical computation performed with

$$
\lambda=1, \quad \mu=0.4, \quad c=4.6 .
$$

The source term $f$ is not compactly supported in $U$ anymore and is given by (see Fig. 6):

$$
f=100(2 x-y) y(1-x) .
$$

We choose the initial domain as the whole domain $U$ without any initial hole. The numerical solution $u$ and its gradient when the optimal domain is reached are represented in Fig. 8. There it can be clearly observed that the Signorini boundary $\Gamma_{S}$ can be divided into two parts: the first part with the homogeneous Dirichlet boundary condition and the second one with the homogeneous Neumann boundary condition. It should be mentioned that the splitting of such boundary conditions cannot be predicted without computations.

Let us point out that the evolution of the domain is very sensitive to small perturbations as we can see from the oscillations of the energy functional during the iterations. This is due to the fact that the shape derivative is only a directional derivative for the nonlinear Signorini 

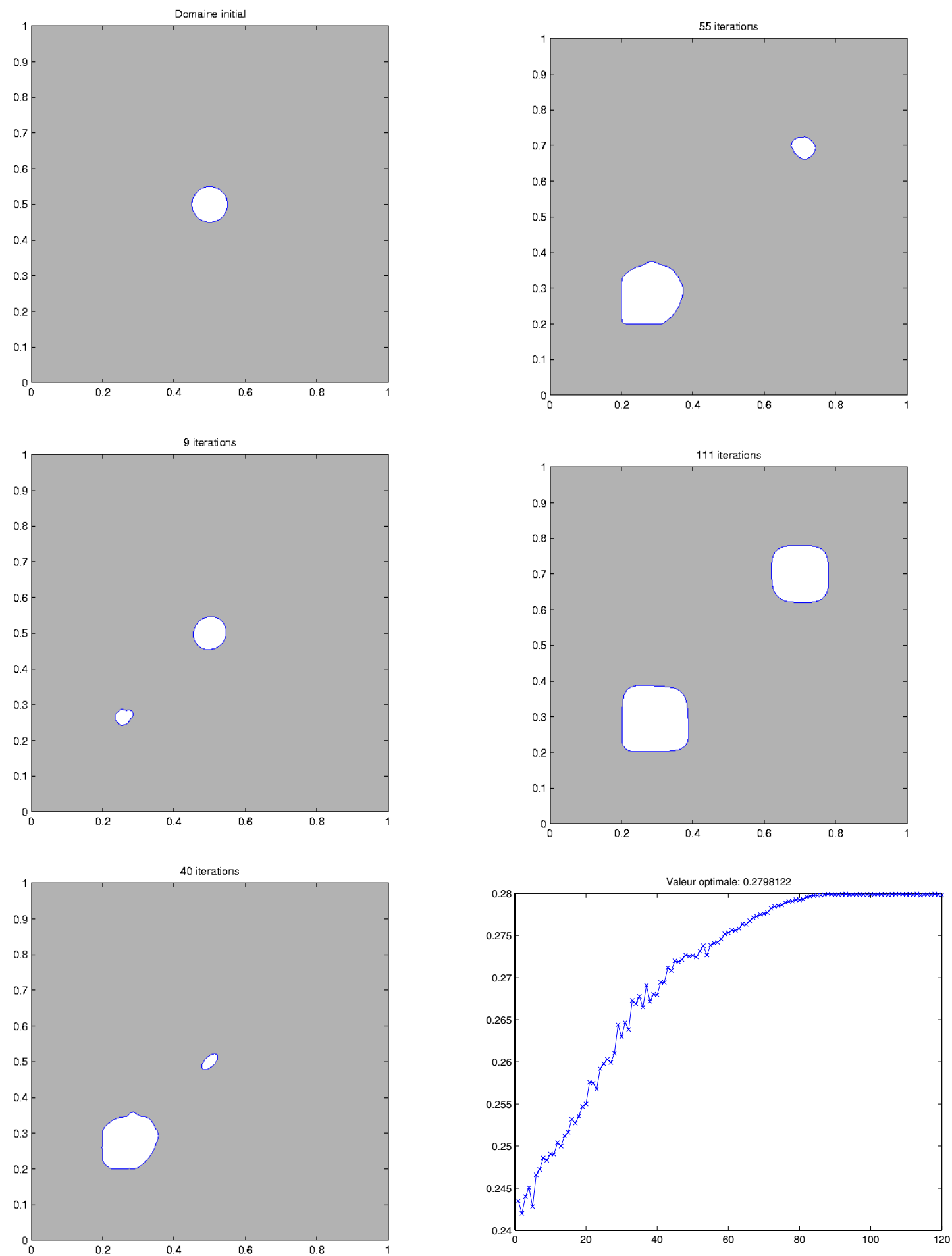

Fig. 5. First numerical example: the evolution of the domain and shape functional $J$.

problem while it is a Fréchet derivative for the related linear problem obtained with $\Gamma_{S}=\emptyset$, i.e., when the external boundary only has Dirichlet boundary conditions.

\section{References}

Allaire G., De Gournay F., Jouve F. and Toader A.M. (2005): Structural optimization using topological and shape sensitivity via a level set method. Control and Cybernetics, Vol. 34, No. 1, pp. 59-80. 

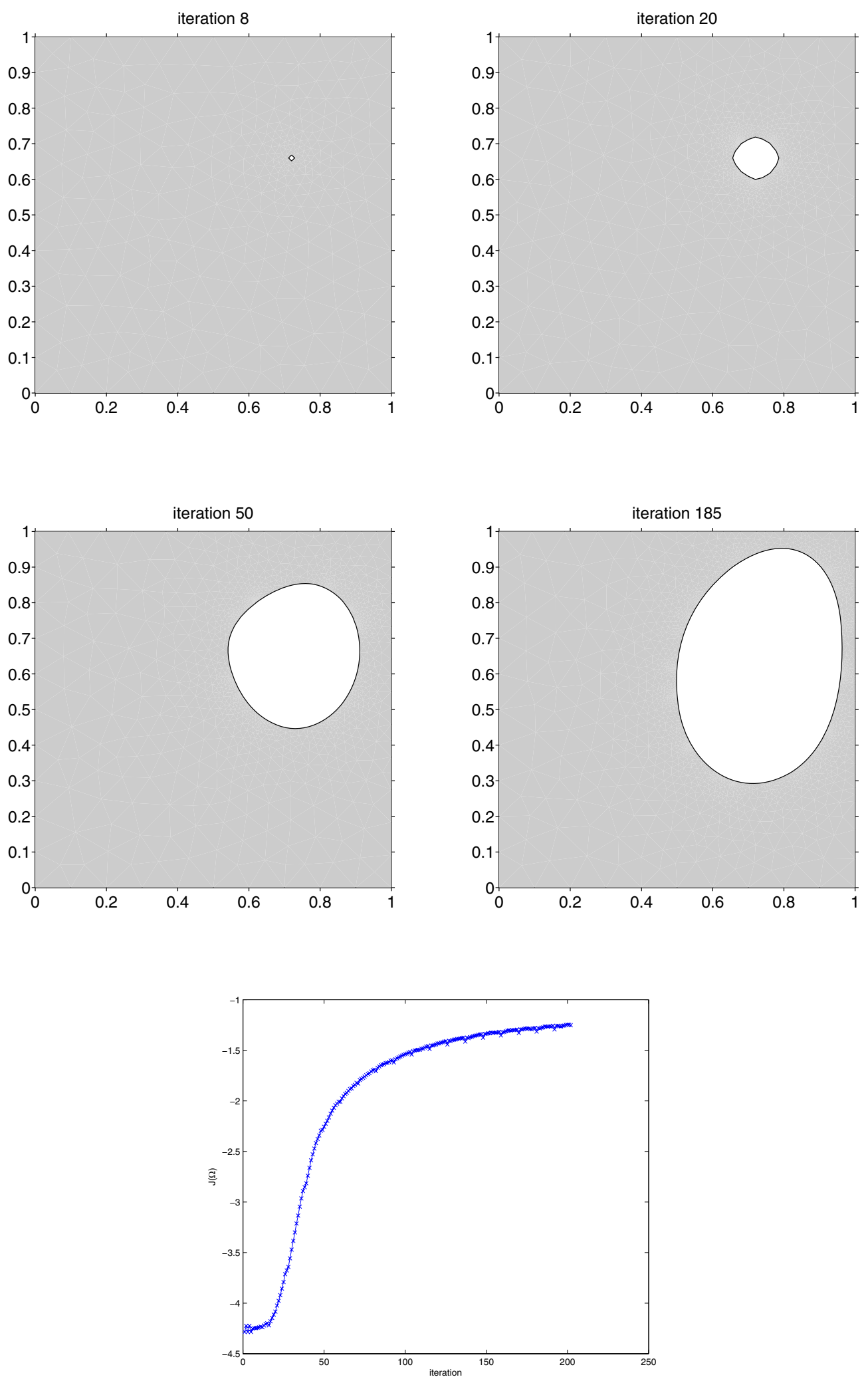

Fig. 7. Second numerical example: the evolution of the domain and shape functional $J$. 


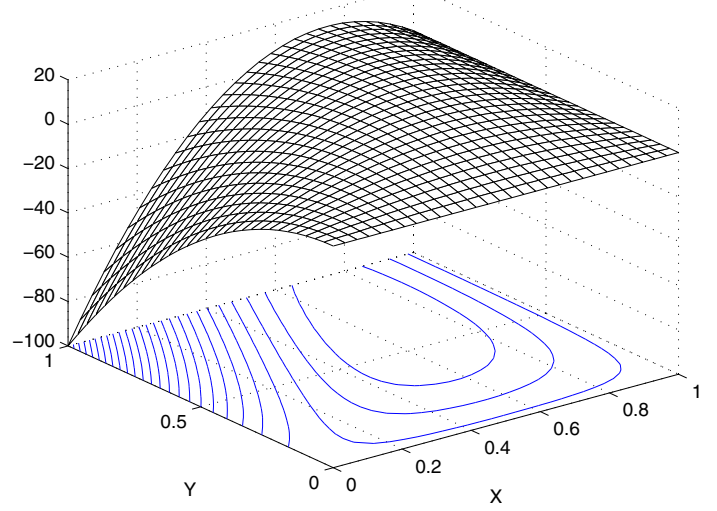

Fig. 6. Second numerical example: the source function $f$.

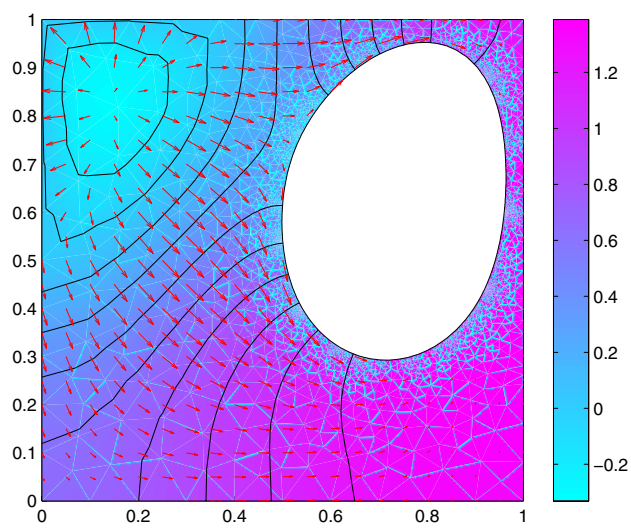

Fig. 8. Second numerical example: the numerical solution $u$ on the optimal domain.

Amstutz S. and Andrä H. (2006): A new algorithm for topology optimization using a level-set method. - Journal of Computer Physics, Vol. 216, No. 2, pp. 573-588.

Delfour M.C. and Zolesio J.-P. (2001): Shapes and Geometries. Philadelphia, PA: SIAM.

Henrot A. and Pierre M. (2005): Variation et optimisation de formes: Une analyse géométrique. Berlin: Springer.

Jackowska L., Sokołowski J., Żochowski A. and Henrot A. (2002): On numerical solution of shape inverse problems. - Computational Optimization and Applications, Vol. 23, No. 2, pp. 231-255.

Jackowska A.L., Sokołowski J. and Żochowski A. (2003): Topological optimization and inverse problems. Computer Assisted Mechanics and Engineering Sciences, Vol. 10, No. 2 , pp. 163-176.

Jarusek J., Krbec M., Rao M. and Sokołowski J. (2003): Conical differentiability for evolution variational inequalities. Journal of Differential Equations, Vol. 193, No. 1, pp. 131146.

Laurain A. (2006): Singularly perturbed domains in shape optimization. - Ph.D. thesis, Université de Nancy.
Masmoudi M. (2002): The topological asymptotic, In: Computational Methods for Control Applications (R. Glowinski, H. Kawarada and J. Periaux, Eds.). GAKUTO Int. Ser. Math. Sci. Appl., Vol. 16, pp. 53-72.

Maz'ya V., Nazarov S.A. and Plamenevskij B. (2000): Asymptotic Theory of Elliptic Boundary Value Problems in Singularly Perturbed Domains. Vols. 1 and 2, Basel: Birkhäuser, p. 435.

Nazarov S.A. (1999): Asymptotic conditions at a point, self adjoint extensions of operators, and the method of matched asymptotic expansions. American Mathematical Society Translations, Vol. 198, No. 2, pp. 77-125.

Nazarov S.A. and Sokołowski J. (2003a): Self adjoint extensions of differential operators in application to shape optimization. Comptes Rendus Mécanique, Vol. 331, No. 10, pp. 667-672.

Nazarov S.A. and Sokołowski J. (2003b): Asymptotic analysis of shape functionals. Journal de Mathématiques pures et appliquées, Vol. 82, No. 2, pp. 125-196.

Nazarov S.A. and Sokołowski J. (2004a): Self adjoint extensions for elasticity system in application to shape optimization. Bulletin of the Polish Academy of Sciences, Mathematics, Vol. 52, No. 3, pp. 237-248.

Nazarov S.A. and Sokołowski J. (2004b): The topological derivative of the Dirichlet integral due to formation of a thin ligament. Siberian Mathematical Journal, Vol. 45, No. 2, pp. 341-355.

Nazarov S.A., Slutskij A.S. and Sokołowski J. (2005): Topological derivative of the energy functional due to formation of a thin ligament on a spatial body. Folia Mathematicae, Acta Universitatis Lodziensis, Vol. 12, pp. 39-72.

Osher S. and Fedkiw R. (2004): Level Set Methods and Dynamic Implicit Surfaces. New York: Springer.

Osher S. and Sethian J. (1988): Fronts propagating with curvature-dependant speed: Algorithms based on Hamilton-Jacobi formulation. Journal of Computational Physics, Vol. 79, No. 1, pp. 12-49.

Peng D., Merriman B., Osher S., Zhao S. and Kang M. (1999): A PDE-based fast local level set method. Journal of Computational Physics, Vol. 155, No. 2, pp. 410-438.

Rao M. and Sokołowski J. (2000): Tangent sets in Banach spaces and applications to variational inequalities. Les prépublications de l'Institut Élie Cartan, No. 42.

Sethian J. (1996): Level Set Methods. Cambridge: Cambridge University Press.

Sokołowski J. and Zolesio J.-P. (1992): Introduction to shape optimization. Series in Computationnal Mathematics, Berlin: Springer Verlag, Vol. 16.

Sokołowski J. and Żochowski A. (1999): On the topological derivative in shape optimization. SIAM Journal on Control and Optimization, Vol. 37, No. 4, pp. 1251-1272.

Sokołowski J. and Żochowski A. (2001): Topological derivatives of shape functionals for elasticity systems. Mechanics of Structures and Machines, Vol. 29, No. 3, pp. 333-351. 
Sokołowski J. and Żochowski A. (2003): Optimality conditions for simultaneous topology and shape optimization. SIAM Journal on Control and Optimization, Vol. 42, No. 4, pp. 1198-1221.

Sokołowski J. and Żochowski A. (2005a): Topological derivatives for contact problems. Numerische Mathematik, Vol. 102, No. 1, pp. 145-179.

Sokołowski J. and Żochowski A. (2005b): Topological derivatives for obstacle problems. Les prépublications de l'Institut Élie Cartan No. 12.

Watson G.N. (1944): Theory of Bessel Functions. Cambridge: The University Press.

Zhao H.K., Chan T., Merriman B. and Osher S. (1996): A variational level set approach to multi-phase motion. Journal of Computational Physics, Vol. 127, No. 1, pp. 179-195.

\section{Appendix}

\section{Topological Derivative with Neumann Conditions on the Hole}

We present results which are used in order to determine the topological derivatives of the energy functional. The technique is proposed in (Masmoudi, 2002; Sokołowski and Żochowski, 2005a). The proof of the asymptotic expansion of the Steklov-Poincaré operator is given in (Sokołowski and Żochowski, 2005b). For the reader's convenience, we present complete arguments.

The domain decomposition technique can be described in the following way. The actual domain is divided into two parts $\Omega_{\rho}=\Omega_{R} \cup \Gamma_{R} \cup C(R, \rho)$. The singular perturbation of the domain is located in the ring $C(R, \rho)$, the moving part of its boundary $\Gamma_{\rho}$ for $\rho>0$ being a small parameter. In the other part of its boundary $\Gamma_{R}$, the Steklov-Poincaré operator is defined and the asymptotics of the operator are determined as a function of the parameter $\rho$. The second domain $\Omega_{R}$ of the decomposition depends only on the parameter $\rho>0$ by the nonlocal boundary conditions prescribed in terms of the Steklov-Poincaré operator $A_{\rho}$, so we have the regular perturbation of the boundary conditions for the nonlinear boundary value problem in $\Omega_{R}$. The conical differentiability of solutions to the variational inequality in $\Omega_{R}$ is shown and results in the asymptotic expansion of the energy functional for our shape optimization problem under investigation.

Now we give the asymptotic expansions of the energy terms $E_{\rho}^{(1)}(v), E_{\rho}^{(2)}(f)$ and $E\left(\Omega_{\rho}, u_{\rho}\right)$ appearing in the relation (23). For simplicity, we shall assume that $x_{0}=0$.

A1. Asymptotic Expansion of $\boldsymbol{E}_{\boldsymbol{\rho}}^{(\mathbf{1})}(\boldsymbol{v})$. To begin with, for any $v$ in $H^{\frac{1}{2}}\left(\Gamma_{R}\right)$, we consider the following problem (see (19)):

$$
\left\{\begin{aligned}
-\Delta w_{\rho}+w_{\rho}=0 & \text { in } C(R, \rho), \\
w_{\rho}=v & \text { on } \Gamma_{R}, \\
\partial_{n} w_{\rho}=0 & \text { on } \Gamma_{\rho} .
\end{aligned}\right.
$$

The Steklov-Poincaré operator $A_{\rho}$ is defined in the following way:

$$
A_{\rho}:\left\{\begin{array}{rlc}
H^{\frac{1}{2}}\left(\Gamma_{R}\right) & \rightarrow & H^{-\frac{1}{2}}\left(\Gamma_{R}\right), \\
v & \mapsto & \partial_{n} w_{\rho} .
\end{array}\right.
$$

Since $v \in H^{\frac{1}{2}}\left(\Gamma_{R}\right)$, we can write $v$ in the form of the Fourier series, with $(r, \phi)$ being the polar coordinates at the origin,

$$
v(\phi)=\frac{1}{2} a_{0}+\sum_{k=1}^{\infty}\left(a_{k} \sin (k \phi)+b_{k} \cos (k \phi)\right) .
$$

The coefficients satisfy

$$
\sum_{k=1}^{\infty} \sqrt{1+k^{2}}\left(a_{k}^{2}+b_{k}^{2}\right) \leq M
$$

where $M$ is a constant depending only on $R$. This implies two important properties:

$$
\sum_{k=1}^{\infty}\left(a_{k}^{2}+b_{k}^{2}\right) \leq M, \quad \sum_{k=1}^{\infty} k\left(a_{k}^{2}+b_{k}^{2}\right) \leq M
$$

The following result gives the asymptotic expansion of the energy term:

$$
E_{\rho}^{(1)}(v)=\int_{C(R, \rho)}\left(\left|\nabla w_{\rho}\right|^{2}+w_{\rho}^{2}\right) \mathrm{d} x
$$

where $w_{\rho}=w_{\rho}(v)$ is the solution to (A1).

Theorem 2. The energy $E_{\rho}^{(1)}(v)$ admits the expansion

$$
\begin{aligned}
E_{\rho}^{(1)}(v)= & E^{(1)}(v)-\left(\frac{\pi\left(a_{1}^{2}+b_{1}^{2}\right)}{2 I_{1}(R)^{2}}+\frac{\pi a_{0}^{2}}{4 I_{0}(R)^{2}}\right) \rho^{2} \\
& +\mathfrak{R}(v),
\end{aligned}
$$

with $E^{(1)}(v)=E_{0}^{(1)}(v)$ and $\mathfrak{R}(v)=o\left(\rho^{2}\right)$ uniformly on bounded subsets of $H^{1}\left(\Omega_{R}\right)$. The Bessel functions $I_{0}$ and $I_{1}$ are defined in (A11).

Proof. Since every compact set can be covered by a finite number of balls, it is enough to prove the lemma for a fixed ball in $H^{1}\left(\Omega_{R}\right)$. Thus we can assume that (A3) occurs. The proof consists in obtaining explicit formulas for $w$ and $w_{\rho}$ in series. Then we can calculate energies explicitly and obtain an upper bound for the remainder $\mathfrak{R}(v)$. 

form

We look for the solution $w_{\rho}$ of (A1) in $C(R, \rho)$ of the

$$
\begin{aligned}
& w_{\rho}(r, \phi) \\
& \qquad \begin{aligned}
2 & \frac{1}{2} a_{0} c_{0, \rho}(r)+\sum_{k=1}^{\infty} c_{k, \rho}(r)\left(a_{k} \sin (k \phi)\right. \\
& \left.+b_{k} \cos (k \phi)\right) .
\end{aligned}
\end{aligned}
$$

The Laplacian in polar coordinates is

$$
\Delta=\frac{\partial^{2}}{\partial r^{2}}+\frac{1}{r} \frac{\partial}{\partial r}+\frac{1}{r^{2}} \frac{\partial}{\partial \phi^{2}} .
$$

Inserting (A5) in $-\Delta w_{\rho}+w_{\rho}=0$, for $k \geq 1$, we obtain

$$
c_{k, \rho}^{\prime \prime}(r)+\frac{1}{r} c_{k, \rho}^{\prime}(r)-\left(\frac{k^{2}}{r^{2}}+1\right) c_{k, \rho}(r)=0,
$$

and

$$
c_{0, \rho}^{\prime \prime}(r)+\frac{1}{r} c_{0, \rho}^{\prime}(r)-c_{0, \rho}(r)=0 .
$$

Multiplying (A6) and (A7) by $r^{2}$, for $k \geq 1$, we get

$$
r^{2} c_{k, \rho}^{\prime \prime}(r)+r c_{k, \rho}^{\prime}(r)-\left(k^{2}+r^{2}\right) c_{k, \rho}(r)=0
$$

and

$$
r^{2} c_{0, \rho}^{\prime \prime}(r)+r c_{0, \rho}^{\prime}(r)-r^{2} c_{0, \rho}(r)=0 .
$$

According to (Watson, 1944), the solutions to (A8) and (A9) are given by

$$
c_{k, \rho}(r)=A_{k} I_{k}(r)+B_{k} K_{k}(r), \quad k \geq 0,
$$

where $A_{k}, B_{k} \in \mathbb{R}$ and with $I_{k}$ and $K_{k}$ being Bessel functions defined by

$$
I_{k}(r)=\sum_{m=0}^{\infty} \frac{\left(\frac{r}{2}\right)^{k+2 m}}{m !(k+m) !}, \quad k \geq 0,
$$

and, for $k \geq 1$,

$$
\begin{aligned}
K_{k}(r)= & \frac{1}{2} \sum_{m=0}^{k-1} \frac{(-1)^{m}(k-m-1) !}{m !\left(\frac{r}{2}\right)^{k-2 m}} \\
& +\sum_{m=0}^{\infty} \frac{(-1)^{k+1}\left(\frac{r}{2}\right)^{k+2 m}}{m !(k+m) !} \tilde{K}_{m}(r)
\end{aligned}
$$

with

$$
\tilde{K}_{m}(r)=\left[\ln \left(\frac{r}{2}\right)-\frac{1}{2} \psi(m+1)-\frac{1}{2} \psi(k+m+1)\right],
$$

and $\psi$ as the logarithmic derivative of function $\Gamma$, i.e.,

$$
\psi(x)=\frac{\partial}{\partial h} \ln \Gamma(x+h) .
$$

Finally, for $k=0$,

$$
K_{0}(r)=-\ln \left(\frac{r}{2}\right) I_{0}(r)+\sum_{m=0}^{\infty} \frac{\left(\frac{r}{2}\right)^{2 m}}{m !^{2}} \psi(m+1) .
$$

The boundary conditions on $\Gamma_{R}$ and $\Gamma_{\rho}$ allow us to obtain the following systems for $k \geq 0$ :

$$
\begin{aligned}
A_{k} I_{k}(R)+B_{k} K_{k}(R) & =1, \\
A_{k} I_{k}^{\prime}(\rho)+B_{k} K_{k}^{\prime}(\rho) & =0 .
\end{aligned}
$$

We deduce the expression of $c_{k, \rho}(r)$ for $k \geq 0$ in a form suited for the asymptotic expansion when $\rho \rightarrow 0$ :

$$
c_{k, \rho}(r)=\frac{I_{k}(r)}{I_{k}(R)}+\dot{c}_{k, \rho}(r)
$$

with

$$
\dot{c}_{k, \rho}(r)=-\frac{\frac{I_{k}^{\prime}(\rho)}{K_{k}^{\prime}(\rho)}}{\frac{I_{k}(R)}{K_{k}(R)}-\frac{I_{k}^{\prime}(\rho)}{K_{k}^{\prime}(\rho)}}\left[\frac{K_{k}(r)}{K_{k}(R)}-\frac{I_{k}(r)}{I_{k}(R)}\right] .
$$

For $k \geq 1$, from (A11) and (A12) we deduce that

$$
\begin{aligned}
I_{k}^{\prime}(\rho) & =\frac{\rho^{k-1}}{2^{k}(k-1) !}+O\left(\rho^{k+1}\right), \\
K_{k}^{\prime}(\rho) & =-\frac{k ! 2^{k-1}}{\rho^{k+1}}+o\left(\rho^{-k-1}\right),
\end{aligned}
$$

so that

$$
\frac{I_{k}^{\prime}(\rho)}{K_{k}^{\prime}(\rho)}-\frac{\rho^{2 k}}{k !(k-1) ! 2^{2 k-1}}+o\left(\rho^{2 k}\right), \quad k \geq 1 .
$$

In particular, we get

$$
\frac{I_{1}^{\prime}(\rho)}{K_{1}^{\prime}(\rho)}=-\frac{\rho^{2}}{2}+o\left(\rho^{2}\right) .
$$

For $k=0$, from (A11) and (A14) we deduce that

$$
I_{0}^{\prime}(\rho)=\frac{\rho}{2}+O\left(\rho^{3}\right), \quad K_{0}^{\prime}(\rho)=-\frac{1}{\rho}+o(\rho) .
$$

Thus we have

$$
\frac{I_{0}^{\prime}(\rho)}{K_{0}^{\prime}(\rho)}=-\frac{\rho^{2}}{2}+o\left(\rho^{2}\right) .
$$

The function $w_{\rho}$ can be extended as

$$
w_{\rho}=w+z_{\rho},
$$

where $w$ is the solution to the problem (A1) for $\rho=0$ given by

$$
\begin{aligned}
w(r, \phi)= & \frac{1}{2} a_{0} \frac{I_{0}(r)}{I_{0}(R)}+\sum_{k=1}^{\infty} \frac{I_{k}(r)}{I_{k}(R)}\left(a_{k} \sin (k \phi)\right. \\
& \left.+b_{k} \cos (k \phi)\right),
\end{aligned}
$$

and

$$
\begin{aligned}
z_{\rho}(r, \phi)= & \frac{a_{0}}{2} \dot{c}_{0, \rho}(r)+\sum_{k=1}^{\infty} \dot{c}_{k, \rho}(r)\left(a_{k} \sin (k \phi)\right. \\
& \left.+b_{k} \cos (k \phi)\right) .
\end{aligned}
$$


Using (A22) in (A4), we get

$$
E_{\rho}^{(1)}(v)=\int_{C(R, \rho)}\left(\left|\nabla w+\nabla z_{\rho}\right|^{2}+\left(w+z_{\rho}\right)^{2}\right) \mathrm{d} x
$$

and then

$$
E_{\rho}^{(1)}(v)=E^{(1)}(v)+I_{1}+I_{2}+I_{3}
$$

with

$$
\begin{aligned}
I_{1}= & \int_{C(R, \rho)}\left(\left(\partial_{r} z_{\rho}\right)^{2}+\frac{1}{r^{2}}\left(\partial_{\phi} z_{\rho}\right)^{2}+z_{\rho}^{2}\right) \mathrm{d} x,(\mathrm{~A} \\
I_{2}= & 2 \int_{C(R, \rho)}\left(\partial_{r} w \partial_{r} z_{\rho}\right. \\
& \left.+\frac{1}{r^{2}} \partial_{\phi} w \partial_{\phi} z_{\rho}+w z_{\rho}\right) \mathrm{d} x \\
I_{3}= & -\int_{B(\rho)}\left(|\nabla w|^{2}+w^{2}\right) \mathrm{d} x
\end{aligned}
$$

We first deal with the integral term $I_{2}$. We have

$$
I_{2}=2 \int_{C(R, \rho)}\left(\left\langle\nabla w, \nabla z_{\rho}\right\rangle+w z_{\rho}\right) \mathrm{d} x .
$$

The function $w$ satisfies $-\Delta w+w=0$ in $C(R, \rho)$. Moreover, according to (A22) and (A1), we have that $z_{\rho}=0$ on $\Gamma_{R}$ and $\partial_{n} w=-\partial_{n} z_{\rho}$ on $\Gamma_{\rho}$. Then we obtain

$$
I_{2}=-2 \int_{\Gamma_{\rho}} z_{\rho} \partial_{n} z_{\rho} \mathrm{d} \sigma .
$$

Since $n$ is the outer normal vector to $C(R, \rho)$, we have $\partial_{n} z_{\rho}=-\partial_{r} z_{\rho}$ on $\Gamma_{\rho}$. Thus, from (A24), and in view of expansions (A16), (A17) and (A20), we can show that the main term in $I_{2}$ is given by

$$
\begin{aligned}
2 \pi \rho\left(a_{1}^{2}+b_{1}^{2}\right) \frac{\mathrm{d} \dot{c}_{1, \rho}}{\mathrm{d} r}(\rho) \dot{c}_{1, \rho}(\rho) & \\
& =-\frac{2 \pi\left(a_{1}^{2}+b_{1}^{2}\right)}{4 I_{1}(R)^{2}} \rho^{2}+o\left(\rho^{2}\right) .
\end{aligned}
$$

Thus we obtain

$$
I_{2}=-\frac{2 \pi\left(a_{1}^{2}+b_{1}^{2}\right)}{4 I_{1}(R)^{2}} \rho^{2}+o\left(\rho^{2}\right) .
$$

Now we turn to the integral $I_{3}$. We have

$$
I_{3}=-\int_{B(\rho)}\left(|\nabla w|^{2}+w^{2}\right) \mathrm{d} x=-\int_{\Gamma_{\rho}} w \partial_{r} w \mathrm{~d} \sigma .
$$

Then it can be shown that

$$
I_{3}=-\left(\frac{\pi a_{0}^{2}}{4 I_{0}(R)^{2}}+\frac{\pi\left(a_{1}^{2}+b_{1}^{2}\right)}{4 I_{1}(R)^{2}}\right) \rho^{2}+o\left(\rho^{2}\right) .
$$

Before calculating $I_{1}$, we will make some remarks. First of all, from (A12), for $k \geq 1$ we have

$$
\int_{\rho}^{R} r K_{k}^{\prime}(r)^{2} \mathrm{~d} r=O\left(\rho^{-2 k}\right)
$$

and for $k=0$ we get

$$
\int_{\rho}^{R} r K_{0}^{\prime}(r)^{2} \mathrm{~d} r=O(\ln \rho)
$$

Then from (A11), for $k \geq 0$

$$
\int_{\rho}^{R} r I_{k}^{\prime}(r)^{2} \mathrm{~d} r=O(1) .
$$

We now split $I_{1}$ into two parts $I_{1}=I_{11}+I_{12}$ with

$$
\begin{aligned}
I_{11} & =\int_{C(R, \rho)}\left(\left(\partial_{r} z_{\rho}\right)^{2}+\frac{1}{r^{2}}\left(\partial_{\phi} z_{\rho}\right)^{2}\right) \mathrm{d} x, \\
I_{12} & =\int_{C(R, \rho)} z_{\rho}^{2} \mathrm{~d} x .
\end{aligned}
$$

As a consequence, since $I_{k}^{\prime}(\rho) / K_{k}^{\prime}(\rho)=O\left(\rho^{2 k}\right)$ for $k \geq 1$ and since $I_{0}^{\prime}(\rho) / K_{0}^{\prime}(\rho)=O\left(\rho^{2}\right)$, the main terms in $I_{11}$ coming from $\left(\partial_{r} z_{\rho}\right)^{2}$ and $\frac{1}{r^{2}}\left(\partial_{\phi} z_{\rho}\right)^{2}$ are respectively given by

$$
\int_{0}^{2 \pi} \int_{\rho}^{R}\left(\frac{\mathrm{d} \dot{c}_{1, \rho}}{\mathrm{d} r}(r)\right)^{2}\left(a_{1}^{2} \sin ^{2}(k \phi)+b_{1}^{2} \cos ^{2}(k \phi) r \mathrm{~d} r \mathrm{~d} \theta\right.
$$

and

$$
\int_{0}^{2 \pi} \int_{\rho}^{R} \frac{1}{r^{2}} \dot{c}_{k, \rho}(r)^{2}\left(a_{1}^{2} \cos ^{2}(k \phi)+b_{1}^{2} \sin ^{2}(k \phi)\right) r \mathrm{~d} r \mathrm{~d} \theta .
$$

The computation of these two terms leads to

$$
I_{11}=\frac{\pi\left(a_{1}^{2}+b_{1}^{2}\right)}{4 I_{1}(R)^{2}} \rho^{2}+o\left(\rho^{2}\right) .
$$

We also show easily that

$$
I_{12}=o\left(\rho^{2}\right)
$$

Now with (A37), (A38), (A32) and (A33) we can conclude that

$$
\begin{aligned}
E_{\rho}^{(1)}(u)=E^{(1)}(v)-\left(\frac{\pi\left(a_{1}^{2}+b_{1}^{2}\right)}{2 I_{1}(R)^{2}}\right. & \left.+\frac{\pi a_{0}^{2}}{4 I_{0}(R)^{2}}\right) \rho^{2} \\
& +o\left(\rho^{2}\right)
\end{aligned}
$$

The proof is thus complete. 
A2. Asymptotic Expansion of $\boldsymbol{E}_{\rho}^{(2)}(f)$. We consider the problem (see (20))

$$
\left\{\begin{aligned}
-\Delta y_{\rho}+y_{\rho} & =f_{\mid C(R, \rho)} & & \text { in } C(R, \rho), \\
y_{\rho} & =0 & & \text { on } \Gamma_{R} \\
\partial_{n} y_{\rho} & =0 & & \text { on } \Gamma_{\rho}
\end{aligned}\right.
$$

with $f \in C^{\infty}\left(\mathbb{R}^{2}\right)$. We shall study the following function:

$$
\left.f_{\mid C(R, \rho)} \mapsto \frac{\partial y_{\rho}}{\partial n}\right|_{\Gamma_{R}}=g_{\rho} .
$$

We would like to obtain an expansion of $g_{\rho}$ with respect to $\rho$. We have the following Fourier expansion for $f$ :

$f(r, \phi)=\frac{1}{2} \tilde{a}_{0}(r)+\sum_{k=1}^{\infty}\left(\tilde{a}_{k}(r) \sin (k \phi)+\tilde{b}_{k}(r) \cos (k \phi)\right)$.

Theorem 3. The function $g_{\rho}$ admits the expansion

$$
\begin{aligned}
g_{\rho}= & g_{0}-\left(\frac{h_{0}^{a}(R)-\tilde{a}_{0}(0)}{4 R I_{0}(R)}\right) \rho^{2} \\
& -\left(\frac{h_{1}^{a}(R)}{2 R I_{1}(R)} \sin \phi+\frac{h_{1}^{b}(R)}{2 R I_{1}(R)} \cos \phi\right) \rho^{2} \\
& +o\left(\rho^{2}\right),
\end{aligned}
$$

where $h_{0}^{a}(R)$ and $h_{1}^{a}(R)$ are defined in (A54) and $h_{1}^{b}(R)$ is obtained from $h_{1}^{a}(R)$ by substituting $\tilde{b}_{1}(t)$ to $\tilde{a}_{1}(t)$ in (A54).

Proof. We look for the solution $y_{\rho}$ of the problem (A40) in the form

$$
y_{\rho}=\frac{1}{2} c_{0, \rho}(r)+\sum_{k=1}^{\infty} c_{k, \rho}(r) \sin (k \phi)+d_{k, \rho}(r) \cos (k \phi) .
$$

Inserting (A42) into $-\Delta y_{\rho}+y_{\rho}=f_{\mid C(R, \rho)}$, we obtain, for $k \geq 0$,

$$
r^{2} c_{k, \rho}^{\prime \prime}(r)+r c_{k, \rho}^{\prime}(r)-\left(k^{2}+r^{2}\right) c_{k, \rho}(r)=-r^{2} \tilde{a}_{k}(r)
$$

and, for $\geq 1$,

$$
r^{2} d_{k, \rho}^{\prime \prime}(r)+r d_{k, \rho}^{\prime}(r)-\left(k^{2}+r^{2}\right) d_{k, \rho}(r)=-r^{2} \tilde{b}_{k}(r) .
$$

Let us deal first with the coefficients $c_{k, \rho}$. We solve (A43) to get (see (Laurain, 2006) for details), for $k \geq 0$,

$$
c_{k, \rho}(r)=A_{k}(r, \rho) I_{k}(r)+B_{k}(r, \rho) K_{k}(r)
$$

with

$$
\begin{aligned}
& A_{k}(r, \rho)=L_{A}(r, k)+\alpha_{k}(\rho), \\
& B_{k}(r, \rho)=L_{B}(r, k)+\beta_{k}(\rho),
\end{aligned}
$$

and

$$
\begin{aligned}
L_{A}(r, k) & =-\int_{R}^{r} t \tilde{a}_{k}(t) K_{k}(t) \mathrm{d} t, \\
L_{B}(r, k) & =\int_{R}^{r} t \tilde{a}_{k}(t) I_{k}(t) \mathrm{d} t .
\end{aligned}
$$

The boundary conditions for $c_{k, \rho}$ are now

$$
c_{k, \rho}(R)=0, \quad c_{k, \rho}^{\prime}(\rho)=0 .
$$

This leads to the following expression for $\alpha_{k}$ and $\beta_{k}$ :

$$
\begin{aligned}
\alpha_{k}(\rho) & =\frac{L_{A}(\rho, k) \frac{I_{k}^{\prime}(\rho)}{K_{k}^{\prime}(\rho)}+L_{B}(\rho, k)}{\frac{I_{k}(R)}{K_{k}(R)}-\frac{I_{k}^{\prime}(\rho)}{K_{k}^{\prime}(\rho)}}, \\
\beta_{k}(\rho) & =-\alpha_{k}(\rho) \frac{I_{k}(R)}{K_{k}(R)} .
\end{aligned}
$$

For $k \geq 2$, the expansions of $\alpha_{k}(\rho)$ and $\beta_{k}(\rho)$ provide terms of orders greater than $\rho^{2}$. Accordingly, we only have to deal with the case $k=1$ and $k=0$.

From (A50) and expansions (A19) and (A21), we get for $i=0,1$

$$
\begin{aligned}
\alpha_{i}(\rho)= & \frac{K_{i}(R)}{I_{i}(R)}\left(L_{A}(\rho, i) \frac{I_{i}^{\prime}(\rho)}{K_{i}^{\prime}(\rho)}+L_{B}(\rho, i)\right) \\
& \times\left(1+\frac{K_{i}(R)}{I_{i}(R)} \frac{I_{i}^{\prime}(\rho)}{K_{i}^{\prime}(\rho)}+O\left(\frac{I_{i}^{\prime}(\rho)^{2}}{K_{i}^{\prime}(\rho)^{2}}\right)\right),
\end{aligned}
$$

and

$$
\begin{aligned}
\alpha_{i}(\rho)= & \alpha_{i}(0)-\frac{K_{i}(R) h_{i}^{a}(R)}{2 I_{i}(R)} \rho^{2} \\
& +\frac{K_{i}(R)}{I_{i}(R)} \int_{0}^{\rho} t \tilde{a}_{i}(t) I_{i}(t) \mathrm{d} t+o\left(\rho^{2}\right),
\end{aligned}
$$

with

$$
\alpha_{i}(0)=-\frac{K_{i}(R)}{I_{i}(R)} \int_{0}^{R} t \tilde{a}_{i}(t) I_{i}(t) \mathrm{d} t
$$

and

$$
h_{i}^{a}(R)=-\frac{K_{i}(R)}{I_{i}(R)} \int_{0}^{R} t \tilde{a}_{i}(t) I_{i}(t) \mathrm{d} t+\int_{0}^{R} t \tilde{a}_{i}(t) K_{i}(t) \mathrm{d} t .
$$

Owing to (A51) we also have the expansion

$$
\beta_{i}(\rho)=\beta_{i}(0)+\frac{h_{i}^{a}(R)}{2} \rho^{2}-\int_{0}^{\rho} t \tilde{a}_{i}(t) I_{i}(t) \mathrm{d} t+o\left(\rho^{2}\right),
$$


with

$$
\beta_{i}(0)=\int_{0}^{R} t \tilde{a}_{i}(t) I_{i}(t) \mathrm{d} t
$$

Here $\beta_{k}(\rho)$ gives us terms of order strictly greater than $\rho^{2}$. In (A52), the term $\int_{0}^{\rho} t \tilde{a}_{i}(t) I_{i}(t) \mathrm{d} t$ gives, for $i=0$,

$$
\int_{0}^{\rho} t \tilde{a}_{0}(t) I_{0}(t) \mathrm{d} t=\frac{\tilde{a}_{0}(0)}{2} \rho^{2}+O\left(\rho^{3}\right) .
$$

On the contrary, for $i=1$, we get due to (A11)

$$
\int_{0}^{\rho} t \tilde{a}_{1}(t) I_{1}(t) \mathrm{d} t=O\left(\rho^{3}\right) .
$$

Thus we have

$$
\alpha_{0}(\rho)=\alpha_{0}(0)-\frac{K_{0}(R)\left(h_{0}^{a}(R)-\tilde{a}_{0}(0)\right)}{2 I_{0}(R)} \rho^{2}+o\left(\rho^{2}\right),
$$

and

$$
\alpha_{1}(\rho)=\alpha_{1}(0)-\frac{K_{1}(R) h_{1}^{a}(R)}{2 I_{1}(R)} \rho^{2}+o\left(\rho^{2}\right) .
$$

Now we can write the expansion of $c_{k, \rho}^{\prime}(R)$ with respect to the small parameter $\rho$. We have

$$
c_{k, \rho}^{\prime}(R)=\alpha_{k}(\rho) I_{k}^{\prime}(R)+\beta_{k}(\rho) K_{k}^{\prime}(R) .
$$

Thus we obtain the expansions

$$
\begin{aligned}
c_{0, \rho}^{\prime}(R)= & c_{0,0}^{\prime}(R)-\frac{h_{0}^{a}(R)-\tilde{a}_{0}(0)}{2} \\
\times & \left(\frac{K_{0}(R)}{I_{0}(R)} I_{0}^{\prime}(R)-K_{0}^{\prime}(R)\right) \rho^{2}+o\left(\rho^{2}\right), \\
c_{1, \rho}^{\prime}(R)= & c_{1,0}^{\prime}(R) \\
& \quad-\frac{h_{1}^{a}(R)}{2}\left(\frac{K_{1}(R)}{I_{1}(R)} I_{1}^{\prime}(R)-K_{1}^{\prime}(R)\right) \rho^{2} \\
& +o\left(\rho^{2}\right),
\end{aligned}
$$

and for $k \geq 2$

$$
c_{k, \rho}^{\prime}(R)=c_{k, 0}^{\prime}(R)+o\left(\rho^{2}\right) .
$$

Finally, we obtain

$$
\begin{aligned}
& c_{0, \rho}^{\prime}(R)=c_{0,0}^{\prime}(R)-\left(\frac{h_{0}^{a}(R)-\tilde{a}_{0}(0)}{2 R I_{0}(R)}\right) \rho^{2}+o\left(\rho^{2}\right), \\
& c_{1, \rho}^{\prime}(R)=c_{1,0}^{\prime}(R)-\frac{h_{1}^{a}(R)}{2 R I_{1}(R)} \rho^{2}+o\left(\rho^{2}\right) .
\end{aligned}
$$

As regards the expansion of $d_{k, \rho}^{\prime}(R)$, we obtain exactly the same results by putting the coefficients $\tilde{a}_{k}(r)$ in place of the coefficients $\tilde{b}_{k}(r)$ in (A61) and (A62). Deriving the Fourier series, we obtain the following expansion:

$$
\begin{aligned}
& \left.\frac{\partial y_{\rho}}{\partial n}\right|_{\Gamma_{R}} \frac{1}{2} c_{0, \rho}^{\prime}(R) \\
& \quad+\sum_{k=1}^{\infty} c_{k, \rho}^{\prime}(R) \sin (k \phi)+d_{k, \rho}^{\prime}(R) \cos (k \phi) .
\end{aligned}
$$

Inserting in (A63) the obtained expansions for $c_{0, \rho}^{\prime}(R)$, $c_{k, \rho}^{\prime}(R)$ and $d_{k, \rho}^{\prime}(R)$, we get

$$
\begin{aligned}
g_{\rho}= & g_{0}-\left(\frac{h_{0}^{a}(R)-\tilde{a}_{0}(0)}{4 R I_{0}(R)}\right) \rho^{2} \\
& -\left(\frac{h_{1}^{a}(R)}{2 R I_{1}(R)} \sin \phi+\frac{h_{1}^{b}(R)}{2 R I_{1}(R)} \cos \phi\right) \rho^{2} \\
& +o\left(\rho^{2}\right) .
\end{aligned}
$$

The expansion (A41) is then proved.

We are now in a position to compute the expansion for the energy term

$$
E_{\rho}^{(2)}(f)=-\int_{C(R, \rho)}\left(\left|\nabla y_{\rho}\right|^{2}+y_{\rho}^{2}\right) \mathrm{d} x .
$$

Theorem 4. The energy $E_{\rho}^{(2)}(f)$ has the expansion

$$
\begin{aligned}
E_{\rho}^{(2)}(f) & \\
& =E^{(2)}(f)-\frac{\pi h_{0}^{a}(R)^{2}}{4} \rho^{2} \\
& \quad-\frac{\pi\left(h_{1}^{a}(R)^{2}+h_{1}^{b}(R)^{2}\right)}{2} \rho^{2}+o\left(\rho^{2}\right) .
\end{aligned}
$$

Proof. Owing to the previous asymptotic expansions, we obtain

$$
\begin{aligned}
c_{0, \rho}(r)= & c_{0,0}(r)-\frac{h_{0}^{a}(R)-\tilde{a}_{0}(0)}{2 I_{0}(R)} M_{0}(r) \rho^{2} \\
& +o\left(\rho^{2}\right), \\
c_{1, \rho}(r)= & c_{1,0}(r)-\frac{h_{1}^{a}(R)}{2 I_{1}(R)} M_{1}(r) \rho^{2}+o\left(\rho^{2}\right),
\end{aligned}
$$

where for $i=0,1, h_{i}^{a}(R)$ is given by (A54) and

$$
\begin{aligned}
c_{0,0}(r)= & K_{0}(r) \int_{0}^{r} t \tilde{a}_{0}(t) I_{0}(t) \mathrm{d} t \\
& -I_{0}(r) \int_{R}^{r} t \tilde{a}_{0}(t) K_{0}(t) \mathrm{d} t \\
& -\frac{K_{0}(R) I_{0}(r)}{I_{0}(R)} \int_{0}^{R} t \tilde{a}_{0}(t) I_{0}(t) \mathrm{d} t, \\
M_{i}(r)= & K_{i}(R) I_{i}(r)-K_{i}(r) I_{i}(R) .
\end{aligned}
$$


For $k \geq 2$, we clearly have

$$
\begin{aligned}
& c_{k, \rho}(r)=c_{k, 0}(r)+o\left(\rho^{2}\right), \\
& d_{k, \rho}(r)=d_{k, 0}(r)+o\left(\rho^{2}\right) .
\end{aligned}
$$

We are able now to give the expansion of the energy. Using the Green formula, we obtain

$$
E_{\rho}^{(2)}(f)=-\int_{C(R, \rho)} f y_{\rho} \mathrm{d} x .
$$

Then we replace $y_{\rho}$ with its expansion in the Fourier series (A42) and we get

$$
\begin{aligned}
E_{\rho}^{(2)}(f) & =\frac{1}{2} \int_{C(R, \rho)} f c_{0, \rho} \mathrm{d} x \\
& -\sum_{k=1}^{\infty} \int_{C(R, \rho)}\left(f c_{k, \rho} \sin (k \phi)+f d_{k, \rho} \cos (k \phi)\right) \mathrm{d} x .
\end{aligned}
$$

From (A65) and substituting $f$ with its expansion in the Fourier series, we have

$$
\begin{aligned}
\int_{C(R, \rho)} f c_{0, \rho} \mathrm{d} x= & \pi \int_{\rho}^{R} r \tilde{a}_{0}(r) c_{0, \rho}(r) \mathrm{d} r \\
= & \pi \int_{\rho}^{R} r \tilde{a}_{0}(r) c_{0,0}(r) \mathrm{d} r \\
& +\frac{\pi\left(h_{0}^{a}(R)^{2}-\tilde{a}_{0}(0) h_{0}^{a}(R)\right)}{2} \rho^{2}+o\left(\rho^{2}\right) \\
= & \pi \int_{0}^{R} r \tilde{a}_{0}(r) c_{0,0}(r) \mathrm{d} r \\
& +\frac{\pi\left(h_{0}^{a}(R)^{2}-2 \tilde{a}_{0}(0) h_{0}^{a}(R)\right)}{2} \rho^{2}+o\left(\rho^{2}\right) .
\end{aligned}
$$

We also have

$$
\begin{aligned}
& \int_{C(R, \rho)} f c_{1, \rho} \sin \phi \mathrm{d} r \\
= & \pi \int_{\rho}^{R} r \tilde{a_{1}}(r) c_{1, \rho}(r) \mathrm{d} r \\
= & \int_{\rho}^{R} r \tilde{a_{1}}(r) c_{1,0}(r) \mathrm{d} r+\frac{\pi h_{1}^{a}(R)^{2}}{2} \rho^{2}+o\left(\rho^{2}\right) \\
= & \pi \int_{0}^{R} r \tilde{a_{1}}(r) c_{1,0}(r) \mathrm{d} r+\frac{\pi\left(h_{1}^{a}(R)^{2}+\tilde{a_{1}}(0) h_{1}^{a}(R)\right)}{2} \rho^{2} \\
& +o\left(\rho^{2}\right) .
\end{aligned}
$$

From the expansion in the Fourier series of $f$, we clearly have $\tilde{a_{1}}(0)=0$. Now owing to (A67) and (A68) we can conclude that

$$
\begin{aligned}
E_{\rho}^{(2)}(f)= & E^{(2)}(f) \\
& -\frac{\pi\left(h_{0}^{a}(R)^{2}-2 \tilde{a}_{0}(0) h_{0}^{a}(R)\right)}{4} \rho^{2} \\
& -\frac{\pi\left(h_{1}^{a}(R)^{2}+h_{1}^{b}(R)^{2}\right)}{2} \rho^{2}+o\left(\rho^{2}\right) .
\end{aligned}
$$

The proof is thus complete.

A3. Asymptotic Expansion of $\boldsymbol{E}\left(\boldsymbol{\Omega}_{\boldsymbol{\rho}}, \boldsymbol{u}_{\boldsymbol{\rho}}\right)$. The energy term $E_{\rho}=E\left(\Omega_{\rho}, u_{\rho}\right)$ is given by (see (23))

$$
\begin{aligned}
E_{\rho}= & -\frac{1}{2} \int_{\Omega_{R}}\left(\left|\nabla u_{\rho}^{R}\right|^{2}+\left(u_{\rho}^{R}\right)^{2}\right) \mathrm{d} x \\
& -\frac{1}{2} E_{\rho}^{(1)}\left(u_{\rho}^{R}\right)+\frac{1}{2} E_{\rho}^{(2)}(f) .
\end{aligned}
$$

Using Theorems 2 and 4, we obtain

$$
\begin{gathered}
E_{\rho}-E_{0} \\
=\int_{\Gamma_{R}} A_{\rho}\left(u_{\rho}^{R}\right) u_{0}^{R} \mathrm{~d} \sigma-\int_{\Gamma_{R}} A_{0}\left(u_{\rho}^{R}\right) u_{0}^{R} \mathrm{~d} \sigma \\
\quad-\frac{\pi\left(h_{0}^{a}(R)^{2}-2 \tilde{a}_{0}(0) h_{0}^{a}(R)\right)}{8} \rho^{2} \\
\quad-\frac{\pi\left(h_{1}^{a}(R)^{2}+h_{1}^{b}(R)^{2}\right)}{4} \rho^{2} \\
\quad+\left(\frac{\pi\left(a_{1}^{2}+b_{1}^{2}\right)}{4 I_{1}(R)^{2}}+\frac{\pi a_{0}^{2}}{8 I_{0}(R)^{2}}\right) \rho^{2} \\
\quad+\int_{\Gamma_{R}} u_{0}^{R} \partial_{n}\left(y_{\rho}-y_{0}\right) \mathrm{d} \sigma+o\left(\rho^{2}\right) .
\end{gathered}
$$

From (A23) and (A24) we have

$$
\begin{aligned}
\int_{\Gamma_{R}} A_{\rho}\left(u_{\rho}^{R}\right) u_{0}^{R} \mathrm{~d} \sigma-\int_{\Gamma_{R}} A_{0}\left(u_{\rho}^{R}\right) u_{0}^{R} \mathrm{~d} \sigma \\
\quad=\int_{\Gamma_{R}}\left(A_{\rho}-A_{0}\right)\left(u_{0}^{R}\right) u_{0}^{R} \mathrm{~d} \sigma+o\left(\rho^{2}\right) \\
=\int_{\Gamma_{R}} w\left(u_{0}^{R}\right) \partial_{n} z_{\rho}\left(u_{0}^{R}\right) \mathrm{d} \sigma+o\left(\rho^{2}\right) \\
=-\frac{\pi a_{0}^{2}}{4 I_{0}(R)^{2}} \rho^{2}-\frac{\pi\left(a_{1}^{2}+b_{1}^{2}\right)}{2 I_{1}(R)^{2}} \rho^{2}+o\left(\rho^{2}\right) .
\end{aligned}
$$

In the previous calculation, we have used the expansion (27). 
Finally, thanks to (A64), we obtain

$$
\begin{aligned}
\int_{\Gamma_{R}} & u_{0}^{R} \partial_{n}\left(y_{\rho}-y_{0}\right) \mathrm{d} \sigma \\
= & \int_{\Gamma_{R}} u_{0}^{R}\left(g_{\rho}-g_{0}\right) \mathrm{d} \sigma \\
= & -\pi\left(\frac{a_{0}(R) h_{0}^{a}(R)-\tilde{a}_{0}(0) a_{0}(R)}{4 I_{0}(R)}+\frac{a_{1} h_{1}^{a}(R)}{2 I_{1}(R)}\right) \rho^{2} \\
& -\pi\left(\frac{b_{1} h_{1}^{b}(R)}{2 I_{1}(R)}\right) \rho^{2}+o\left(\rho^{2}\right) .
\end{aligned}
$$

Finally, with the previous expansions and noticing that $\tilde{a}_{0}(0)=2 f(0)$, we obtain the following result:

Theorem 5. The energy $E\left(\Omega_{\rho}, u_{\rho}\right)$ admits the following expansion:

$$
\begin{aligned}
E\left(\Omega_{\rho}, u_{\rho}\right) & \\
=E & (\Omega, u) \\
- & {\left[\frac{a_{0}(R)^{2}}{8 I_{0}(R)^{2}}+\frac{h_{0}^{a}(R)^{2}}{8}+\frac{a_{0}(R) h_{0}^{a}(R)}{4 I_{0}(R)}\right] \pi \rho^{2} } \\
- & {\left[\frac{a_{1}(R)^{2}+b_{1}(R)^{2}}{4 I_{1}(R)^{2}}\right] \pi \rho^{2} } \\
- & {\left[\frac{a_{1}(R) h_{1}^{a}(R)}{2 I_{1}(R)}+\frac{b_{1}(R) h_{1}^{b}(R)}{2 I_{1}(R)}\right] \pi \rho^{2} } \\
- & {\left[\frac{h_{1}^{a}(R)^{2}}{4}+\frac{h_{1}^{b}(R)^{2}}{4}\right] \pi \rho^{2} } \\
+ & {\left[\frac{f(0) a_{0}(R)}{2 I_{0}(R)}+\frac{f(0) h_{0}^{a}(R)}{2}\right] p_{i} \rho^{2}+o\left(\rho^{2}\right) . }
\end{aligned}
$$

The coefficients $a_{0}, a_{1}$ and $b_{1}$ are given by

$$
\begin{aligned}
& a_{0}(R)=\frac{1}{\pi} \int_{0}^{2 \pi} u(R, \phi) d \phi, \\
& a_{1}(R)=\frac{1}{\pi} \int_{0}^{2 \pi} u(R, \phi) \sin \phi d \phi, \\
& b_{1}(R)=\frac{1}{\pi} \int_{0}^{2 \pi} u(R, \phi) \cos \phi d \phi .
\end{aligned}
$$

The functions $h_{i}^{a}(R), i=0,1$ are defined in (A54).

The quantities between brackets in (A71) do not depend on $R$, and we can easily show that

$$
u(0)=\frac{a_{0}(R)}{2 I_{0}(R)}+\frac{h_{0}^{a}(R)}{2},
$$

and therefore

$$
\frac{u(0)^{2}}{2}=\frac{a_{0}(R)^{2}}{8 I_{0}(R)^{2}}+\frac{h_{0}^{a}(R)^{2}}{8}+\frac{a_{0}(R) h_{0}^{a}(R)}{4 I_{0}(R)} .
$$

Moreover,

$$
\begin{aligned}
& |\nabla u(0)|^{2} \\
& \qquad \begin{array}{l}
a_{1}(R)^{2}+b_{1}(R)^{2} \\
4 I_{1}(R)^{2}
\end{array}+\frac{a_{1}(R) h_{1}^{a}(R)}{2 I_{1}(R)}+\frac{b_{1}(R) h_{1}^{b}(R)}{2 I_{1}(R)} \\
& \quad+\frac{h_{1}^{a}(R)^{2}}{4}+\frac{h_{1}^{b}(R)^{2}}{4} .
\end{aligned}
$$

We then deduce a different expression of the previous asymptotic expansion, which actually leads to the usual expression for the topological derivative

$$
\begin{aligned}
E\left(\Omega_{\rho}, u_{\rho}\right)= & E(\Omega, u) \\
& +\left[-\frac{u(0)^{2}}{2}-|\nabla u(0)|^{2}+f(0) u(0)\right] \pi \rho^{2} \\
& +o\left(\rho^{2}\right) .
\end{aligned}
$$

Let us mention that for $i=0,1$

$$
\begin{aligned}
& \lim _{R \rightarrow 0} h_{i}^{a}(R)=0, \\
& \lim _{R \rightarrow 0} h_{1}^{b}(R)=0,
\end{aligned}
$$

and, therefore, (A71) gives an approximation of the topological derivative that can be calculated on the curve $\Gamma_{R}$, which can be interesting from a numerical point of view. In particular, it is possible to compute $a_{1}(R)$ and $b_{1}(R)$ without computing the gradient of the solution $u$. 